\title{
Neonatal Systemic Exposure to Lipopolysaccharide Enhances Susceptibility of Nigrostriatal Dopaminergic Neurons to Rotenone Neurotoxicity in Later Life
}

\author{
Zhengwei Cai $^{\mathrm{a}}$ Lir-Wan Fan ${ }^{\mathrm{a}}$ Asuka Kaizaki ${ }^{\mathrm{c}}$ Lu-Tai Tien ${ }^{\mathrm{d}}$ Tangeng Ma ${ }^{\mathrm{a}}$ \\ Yi Pang ${ }^{a}$ Shuying Lin ${ }^{a}$ Rick C.S. Lin ${ }^{b}$ Kimberly L. Simpson ${ }^{b}$ \\ ${ }^{a}$ Division of Newborn Medicine, Department of Pediatrics, and ${ }^{b}$ Department of Neurobiology and \\ Anatomical Sciences, University of Mississippi Medical Center, Jackson, Miss., USA; ' $D e p a r t m e n t ~ o f$ \\ Biochemical Toxicology, School of Pharmacy, Showa University, Tokyo, Japan; ${ }^{d}$ School of Medicine, \\ Fu Jen Catholic University, New Taipei City, Taiwan, ROC
}

\section{Key Words}

Neonatal brain injury · Neuroinflammation .

Lipopolysaccharide $\cdot$ Substantia nigra $\cdot$ Tyrosine

hydroxylase - Mitochondrial injury - Secondary injury .

Rotenone · Parkinson's disease

\begin{abstract}
Brain inflammation via intracerebral injection with lipopolysaccharide (LPS) in early life has been shown to increase risks for the development of neurodegenerative disorders in adult rats. To determine if neonatal systemic LPS exposure has the same effects on enhancement of adult dopaminergic neuron susceptibility to rotenone neurotoxicity as centrally injected LPS does, LPS ( $2 \mu \mathrm{g} / \mathrm{g}$ body weight) was administered intraperitoneally into postnatal day 5 (P5) rats and when grown to P70, rats were challenged with rotenone, a commonly used pesticide, through subcutaneous minipump infusion at a dose of $1.25 \mathrm{mg} / \mathrm{kg} /$ day for 14 days. Systemically administered LPS can penetrate into the neonatal rat brain and cause acute and chronic brain inflammation, as evidenced by persistent increases in IL-1 $\beta$ levels, cyclooxygenase- 2 expression and microglial activation in the sub-
\end{abstract}

stantia nigra (SN) of P70 rats. Neonatal LPS exposure resulted in suppression of tyrosine hydroxylase (TH) expression, but not actual death of dopaminergic neurons in the $\mathrm{SN}$, as indicated by the reduced number of $\mathrm{TH}+$ cells and unchanged total number of neurons (NeuN+) in the SN. Neonatal LPS exposure also caused motor function deficits, which were spontaneously recoverable by P70. A small dose of rotenone at P70 induced loss of dopaminergic neurons, as indicated by reduced numbers of both $\mathrm{TH}+$ and $\mathrm{NeuN}+$ cells in the $\mathrm{SN}$, and Parkinson's disease (PD)-like motor impairment in P98 rats that had experienced neonatal LPS exposure, but not in those without the LPS exposure. These results indicate that although neonatal systemic LPS exposure may not necessarily lead to death of dopaminergic neurons in the $\mathrm{SN}$, such an exposure could cause persistent functional alterations in the dopaminergic system and indirectly predispose the nigrostriatal system in the adult brain to be damaged by environmental toxins at an ordinarily nontoxic or subtoxic dose and develop PD-like pathological features and motor dysfunction.
Copyright $\odot 2013$ S. Karger AG, Basel

\section{KARGER}

E-Mail karger@karger.com

www.karger.com/dne
(C) 2013 S. Karger AG, Basel

0378-5866/13/0353-0155\$38.00/0
Dr. Zhengwei Cai, $\mathrm{PhD}$

Department of Pediatrics, Division of Newborn Medicine

University of Mississippi Medical Center

Jackson, MS 39216-4504 (USA)

E-Mail zcai@umc.edu 


\section{Introduction}

Increasing evidence has indicated that perinatal infection/inflammation not only is a major contributor to brain injury in newborns, but also has long-term consequences and could speculatively modify the risk of a variety of neurological disorders in children and adults [1]. Animal studies have shown that neuropsychiatric disorders such as schizophrenia and autism spectrum disorders in children and adults are possibly linked with prenatal infection/inflammation [2-4]. Maternal injection of an endotoxin, lipopolysaccharide (LPS), has recently been reported to impair fetal brain development in rats shortly after the exposure [5] and to alter the neuroimmune response in adult offspring [6]. Although the detailed mechanisms involved remain unclear, it has also been proposed that early-life occurrence of inflammation in the brain, as a consequence of either brain injury or exposure to infectious agents, may play a role in the pathogenesis of Parkinson's disease (PD) [7, 8], a neurodegenerative disease typically seen in aged people. Prenatal or early-life exposure to LPS has been shown to increase the risk of dopaminergic disorders in animal models of PD [9-11]. In utero exposure to LPS in rats was found to be associated with reduced number of dopaminergic neurons in the substantia nigra (SN) of adult brain and possibly to contribute to PD $[10,12]$. However, in utero LPS exposure does not produce an accelerated rate of dopaminergic neuron loss [13], rather, progressive dopaminergic neuron loss in rats exposed to LPS prenatally requires a second dose of LPS challenge [11]. It is unclear whether death of dopaminergic neurons results from events initiated during development, adulthood, or represents a cumulative effect across the life span $[14,15]$.

Our recent studies have shown that neonatal exposure [postnatal day 5 (P5)] to LPS through an intracerebral injection in rats can produce injury in the nigrostriatal dopaminergic system, as indicated by the phenotypic suppression of tyrosine hydroxylase $(\mathrm{TH})$ expression from neurons in the SN and neurobehavioral deficits in the LPS-exposed rats [16-19]. However, neonatal LPS exposure did not cause actual death of dopaminergic neurons in the SN and the LPS-induced motor dysfunction was spontaneously recoverable by adult ages (P70) [18, 19]. On the other hand, when $P 70$ rats were challenged with rotenone, a pesticide used to induce neuropathological features of PD in animal models [20-23], an ordinarily nontoxic or subtoxic dose of rotenone caused actual dopaminergic neuron loss in the SN and PD-like motor dysfunctions in adult rats that had been exposed to
LPS on P5, but not in those without the LPS injection [19]. These findings suggest that perinatal exposure to LPS may not directly cause death of dopaminergic neurons, but may indirectly predispose the nigrostriatal system in the adult brain to be damaged by environmental toxins at an ordinarily nontoxic or subtoxic dose. At present, the etiology of sporadic PD remains largely unknown. The 'multiple hit' hypothesis is a currently well-accepted putative mechanism for sporadic PD [24]. Our findings provide supportive evidence for the 'multiple hit' concept. One caveat of our previous study is that the neonatal LPS exposure was achieved through intracerebral injection, a scenario rarely happening in human life. In the present study, we change the route of neonatal LPS exposure to intraperitoneal injection, a more pathophysiologically relevant approach. The ultimate objective of this study is to determine if neonatal systemic LPS exposure will also enhance adult susceptibility to the development of neurodegenerative disorders triggered by environmental toxins at an ordinarily nontoxic or subtoxic dose, as centrally injected LPS does.

\section{Methods}

\section{Chemicals}

Unless otherwise stated, all chemicals used in this study were purchased from Sigma (St. Louis, Mo., USA). Monoclonal mouse antibodies against neuron-specific nuclear protein (NeuN), phospho-p38 mitogen-activated protein kinase (p-p38 MAPK) or OX42 (CD11b) were purchased from Millipore (Billerica, Mass., USA), Cell Signaling (Danvers, Mass., USA) and Serotec (Raleigh, N.C., USA), respectively. Polyclonal rabbit antibodies against TH or p38 MAPK were purchased from Millipore and Cell Signaling, respectively. Polyclonal goat antibody against cyclooxygenase-2 (COX-2) was obtained from Santa Cruz Biotechnology (Santa Cruz, Calif., USA). ELISA kits for the detection of rat TNF- $\alpha$, IL$1 \beta$ or IL-6, or LPS were obtained from R\&D Systems (Minneapolis, Minn., USA) and MyBiosource (San Diego, Calif., USA), respectively. Fluorogold (FG) for retrograde study was purchased from Fluorochrome (Denver, Colo., USA).

\section{Animals and LPS Exposure}

Timed pregnant Sprague-Dawley rats arrived in the laboratory on day 19 of gestation. Animals were maintained in a room with a 12-hour light/12-hour dark cycle and at constant temperature (22 $\pm 2{ }^{\circ} \mathrm{C}$ ). The day of birth was defined as P0. After birth, the litter size was adjusted to 12 pups per litter to minimize the effect of litter size on body weight and brain size. In order to eliminate a possible gender difference, only male rats were used in the present study. Intraperitoneal injection of LPS (from Escherichia coli, serotype 055:B5) was performed in 5-day-old male Sprague-Dawley rat pups. In our initial studies, rat pups were injected with LPS at a variety of doses $(0-3.0 \mu \mathrm{g} / \mathrm{g}$ or $0-9,000 \mathrm{EU} / \mathrm{g}$ body weight) in sterile saline (total volume of $100 \mu \mathrm{l}$ ) to determine the dose-re- 
sponse relationship (see results, fig. 2), and it was administered intraperitoneally at a dose of $2 \mu \mathrm{g} / \mathrm{g}$ body weight in all other studies. The control rats were injected with the same volume of sterile saline. All animals survived the injection. Each dam had the same litter size (12 pups), and equal numbers of LPS-treated and salinetreated rat pups were included in a litter. The pups were weaned at P21 and 4 rats (2 LPS-treated and 2 saline-treated) per cage were housed thereafter. All procedures for animal care were conducted in accordance with the National Institutes of Health Guide for the Care and Use of Laboratory Animals and were approved by the Institutional Animal Care and Use Committee at the University of Mississippi Medical Center. Every effort was made to minimize the number of animals used and their suffering.

\section{Minipump Preparation and Implantation}

Rotenone was administered through a subcutaneous osmotic minipump infusion as described previously [19]. Briefly, rats pretreated with LPS or saline on P5 were randomly further divided into two groups at P70: one group received rotenone infusion and the other group received vehicle infusion. Thus, 4 experimental groups (12 male rats for each group) were included in the rotenone challenge study: saline/vehicle, saline/rotenone, LPS/vehicle and LPS/rotenone. Alzet osmotic minipumps (2ML4, Durect Corp., Cupertino, Calif., USA) were aseptically filled 1 day before implantation with rotenone in equal amounts of dimethyl sulfoxide and polyethylene glycol 300 (1:1) or with dimethyl sulfoxide/polyethylene glycol 300 vehicle alone. The filled minipumps were placed into sterile $0.9 \%$ saline at $37^{\circ} \mathrm{C}$ overnight until implantation. Under anesthesia with $2 \%$ isoflurane in oxygen, minipumps were placed into subcutaneous burrows on the dorsal surface for infusion. The pumps were designed to deliver $1.25 \mathrm{mg}$ rotenone $/ \mathrm{kg} /$ day or vehicle for 14 days (from P70 to P84). At the end of 14 days (P84), the pumps were removed and the skin was closed with wound clips. Animals were allowed to recover for an additional 14 days (from P85 to P98) to provide sufficient time for the development of a stable lesion.

Twelve rats from each group were used in the rotenone challenge study. Behavioral tests were conducted weekly in these rats from each group from P70 to P98. Rats were sacrificed on P98 by transcardiac perfusion with regular saline followed by $4 \%$ paraformaldehyde for brain section preparation. In addition to these 48 rats, 36 rat pups were used for the initial dose-response study and the LPS brain entry study, 30 P6 rats and 48 P70 from the LPS- and saline-treated group were sacrificed by decapitation or by transcardiac perfusion for fresh brain tissue collection (10 rats from each group for determination of the mitochondrial complex I activity and Western blot analysis) or for brain section preparation (5 rats from each group). The remaining P70 rats ( 9 from each group) were used for electron microscope (EM) study (4 rats from each group) or for FG retrograde study (5 rats from each group). The total number of animals used in this study was 162 male rats from 30 litters.

\section{Immunohistochemistry}

For brain section preparation, rats were sacrificed by transcardiac perfusion with normal saline followed by $4 \%$ paraformaldehyde and free-floating coronal brain sections at $40 \mu \mathrm{m}$ of thickness were prepared in a freezing microtome (Leica, SM 2000R, Wetzlar, Germany). Primary antibodies were used in the following dilutions: NeuN (1:200), TH (1:1,000), COX-2 (1:100) and OX42 (1:200).
NeuN detects the neuron-specific nuclear protein which primarily localizes in the nucleus of the neurons with slight staining in the cytoplasm. TH was used to detect dopaminergic neurons in SN. COX2 provides selective staining of inducible cyclooxygenase. Microglia were detected using OX42 immunostaining, which recognizes both the resting and the activated microglia. Sections were incubated with primary antibodies at $4{ }^{\circ} \mathrm{C}$ overnight and further incubated with secondary antibodies conjugated with fluorescent dyes (Alexa Fluor 555, 1:500 or Alexa Fluor 488, 1:200; Invitrogen, Carlsbad, Calif., USA) for $1 \mathrm{~h}$ in the dark at room temperature. Sections incubated in the absence of primary antibody were used as negative controls. The resulting sections were examined under a fluorescent microscope (Olympus, BX60) at appropriate wavelengths.

\section{Surgical Procedure of Retrograde Labeling}

Surgical procedure of retrograde study was performed as previously described [25]. Under light anesthesia with isoflurane (1.5\%), 5 P70 rats from the LPS-exposed or the control group were placed in a stereotaxic apparatus, a dental drill was used to make a small craniotomy in the skull, and the retrograde tracer $(0.6 \mu$ of FG, $5 \%$ solution in distilled water, Fluorochrome Inc.) was stereotaxically injected into the caudate-putamen bilaterally at the following coordinates relative to bregma: $+0.7 \mathrm{~mm}$ anteroposterior, $\pm 3 \mathrm{~mm}$ mediolateral, and $-5 \mathrm{~mm}$ dorsoventral. The mechanical injection with a $10-\mu \mathrm{l}$ Hamilton syringe lasted for a period of $20 \mathrm{~min}$. Leakage from the tip of the syringe was avoided by introducing a small air bubble toward the opening of the needle just after the tracer was drawn. After a 4-day survival period, animals were transcardiacally perfused with saline followed by $3.5 \%$ paraformaldehyde. The brains were postfixed in the same fixative overnight at $4^{\circ} \mathrm{C}$ and then transferred to a $20 \%$ sucrose/phosphate cryoprotecting solution. Retrogradely labeled FG+ neurons were examined in the coronal brain section $(40 \mu \mathrm{m})$ by UV illumination in a series of sections in combination with stereological cell counting of doublelabeled $\mathrm{NeuN}+$ and $\mathrm{TH}+$ neurons.

\section{Stereological Estimates of the Total Number of Neurons in} the SN

The stereological estimates of the total number of $\mathrm{TH}+$ and $\mathrm{NeuN}+$ neurons, and of COX-2+ neurons (est $\mathrm{N}$ ) were performed in the SN of P70 and P98 rat brains, respectively, following the methods described by Ling et al. [26] and Lokkegaard et al. [27]. Nine equally spaced sections $(40 \mu \mathrm{m})$ in the midbrain level that were to be used in the analysis came from a 1-in- 6 series. The total number of $\mathrm{TH}+, \mathrm{NeuN}+$ or $\mathrm{COX}-2+$ cells (est $N$ ) was counted in each of the 9 sections, which cover the entire SN region. Our preliminary study showed that there were no differences in the density of $\mathrm{TH}+$ or NeuN+ cells between the left and right sides of the rat brain. Therefore, stereological cell counting in the present study was conducted only in the left $\mathrm{SN}$ of the rat brain. It has been reported that the loss of TH-immunoreactive cells caused by prenatal LPS exposure or combined prenatal LPS exposure with postnatal rotenone exposure in rats was primarily observed in the lateral subregion of the SN [26]. Thus, stereological cell counting in the present study was focused on the SN region (nucleus A9 cells or cells in both the SN pars compacta and the SN pars reticulata). The Cavalieri principle [28] was used to estimate the reference volumes, est $V(r e f)$, and the volume density, est $N_{V}$. The product of the two is an estimate of the total number of cells in this region: est $V($ ref $) \times$ est $N_{V}=$ est $N[26,27,29]$. 


\section{Brain Sample Preparation for EM Study}

Brain samples for the EM study were prepared following the procedure described previously [19]. Briefly, 4 P70 rats from each group were transcardiacally perfused with saline followed by $3 \%$ paraformaldehyde plus $0.5 \%$ glutaraldehyde in $0.1 \mathrm{M}$ PBS. Brains were postfixed in the same fixative overnight at $4^{\circ} \mathrm{C}$ and then cut coronally into 50 - to $100-\mu \mathrm{m}$ sections with a vibratome (Lancer). A small area/block (approx. $1 \times 1 \mathrm{~mm}$ ) in the $\mathrm{SN}$ was dissected out with a No. 10 blade. Typically, 2-3 blocks were dissected from each brain. Blocks were processed using standard EM osmication with en bloc staining procedures, flat embedded in EPON, attached to beam capsules, trimmed and cut into ultrathin sections. Sections were collected onto grids coated with Formvar, and then further stained with lead citrate and uranyl acetate. Materials were examined and photographed with a Leo Biological transmission EM by an investigator blinded to the treatment.

\section{Determination of Mitochondrial Complex I Activity}

Complex I activity was determined by a spectrophotometric assay as previously described [19], based on the quantification of the rate of oxidation of the complex I substrate NADH to ubiquinone $[30,31]$. Rats were sacrificed by decapitation 1 day (P6) or 65 days (P70) after the LPS injection, and bilateral regions of the striatum (ST), SN and ventral tegmental area were isolated, frozen in liquid nitrogen, and stored at $-80^{\circ} \mathrm{C}$. Brain tissues were homogenized in $10 \mathrm{~mm}$ Tris-HCl buffer ( $\mathrm{pH} 7.2$ ), containing $225 \mathrm{~mm}$ mannitol, $75 \mathrm{mM}$ saccharose and $0.1 \mathrm{mM}$ EDTA, sonicated on ice, and centrifuged at $4^{\circ} \mathrm{C}(600 \mathrm{~g}, 20 \mathrm{~min})$. The optical density of the supernatants ( $40 \mu \mathrm{g}$ sample protein) in $1 \mathrm{ml}$ of an assay mixture was spectrophotometrically recorded at a wavelength of $340 \mathrm{~nm}$ for $200 \mathrm{~s}$ at $37^{\circ} \mathrm{C}$. The assay mixture was a potassium phosphate buffer (25 mM, pH 7.5) containing $2 \mathrm{~mm}$ potassium cyanide, $5 \mathrm{~mm}$ magnesium chloride, $2.5 \mathrm{mg} / \mathrm{ml}$ bovine serum albumin, $2 \mu \mathrm{M}$ antimycin $\mathrm{A}, 100 \mu \mathrm{M}$ decylubiquinone and $300 \mu \mathrm{M}$ NADH. The proportion of NADH oxidation sensitive to an excess of rotenone $(10 \mu \mathrm{M})$ was attributed to the complex I. The specific activity (nmol NADH oxidation/min/mg protein) of complex I (NADH-ubiquinone oxidoreductase) was calculated using a molar extinction coefficient $\varepsilon_{340 \mathrm{~nm}}=6.22 \mathrm{mM}^{-1} \mathrm{~cm}^{-1}$ [32]. Enzyme activities were expressed as nanomoles per minute per milligram of brain tissue: complex I activity $=\left[\right.$ rate $\left.\left(\mathrm{min}^{-1}\right) / \varepsilon_{340 \mathrm{~nm}}\left(6.22 \mathrm{mM}^{-1} \mathrm{~cm}^{-1}\right)\right] / 0.040 \mathrm{mg}$.

\section{Immunoblotting and ELISA}

Protein expression of TH, p38 MAPK and p-p38 MAPK was determined in the P6 rat brain and the SN and the ST of P70 rat brain by Western blotting. Brain tissues were homogenized in an extraction buffer (Biosource, Camarillo, Calif., USA) added with a mixture of protease inhibitors (Calbiochem, La Jolla, Calif., USA) and $1 \mathrm{mM}$ PMSF. Protein levels of homogenates were determined by the Bradford method. The homogenates were diluted with 1:2 $(\mathrm{v} / \mathrm{v})$ Laemmli sample buffer plus $5 \%(\mathrm{w} / \mathrm{v}) \beta$-mercaptoethanol and boiled for $5 \mathrm{~min}$. Sodium dodecyl sulfate-polyacrylamide gel electrophoresis on a $3.75 \%$ stacking/10\% running gel was performed with an equal amount of protein from each sample $(20$ $\mu \mathrm{g} / 10 \mu \mathrm{l})$. The separated proteins were transferred electrophoretically to PVDF membranes (Bio-Rad Laboratories, Hercules, Calif., USA) at $100 \mathrm{~V}$ for $1 \mathrm{~h}$. The blots were incubated with a blocking solution containing $5 \%$ non-fat milk and $0.1 \%$ Tween-20 in Trisbuffered saline for $1 \mathrm{~h}$ before incubation with the primary antibody $(\mathrm{TH}, 1: 4,000$; 38 or p-p38, 1:1,000) in the blocking solution over- night at $4{ }^{\circ} \mathrm{C}$. The blots were then incubated with peroxidase-conjugated antibodies in the blocking solution $(1: 4,000)$ for $1 \mathrm{~h}$ at room temperature. The immunoreactivity was detected by the Enhanced Chemiluminescence Plus or advanced ECL system (GE Healthcare, Piscataway, N.J., USA) and then determined with the Molecular Imager ${ }^{\circledR}$ ChemiDoc $^{\mathrm{TM}} \mathrm{XRS}+$ system followed by quantification using Quantity One software (both from Bio-Rad Laboratories). To ensure that equal amounts of protein were applied to the immunoblot, the membranes were stripped with a stripping buffer (Thermo Scientific, Rockford, Ill., USA) and reprobed for $\beta$-actin $(1: 4,000)$ to normalize the results.

Protein expression of TNF- $\alpha$, IL- $1 \beta$ and IL- 6 in the P6 rat brain and the SN and ST of P70 rat brain as well as the concentration of LPS in the rat brain were determined by ELISA kits as described previously [18] or following the manufacturer's instruction (LPS).

\section{Behavioral Testing}

Behavioral tests sensitive to varying degrees of dopamine loss in the ST and SN were used in this study to assess motor function $[21,33]$. These tests included the vibrissa-elicited forelimb-placing test, pole test, and tapered/ledged beam walking test. All animals were tested and videotaped in the same order once a week from P14 to P98 by a person blinded to treatment groups. Since recovery or development of lesion following rotenone administration may vary with time, behavioral tests were performed on the same day for all animals, i.e. P70, P77, P84, P91 and P98. Details of these tests have been described in our previous study [18].

Vibrissa-Elicited Forelimb-Placing Test

This test is used to measure forelimb placing deficit upon stimulation of the rat's vibrissae to trigger a placing response [34, 35]. Rats use their vibrissae to gain bilateral information about the proximal environment and this information is integrated between the hemispheres. In the cross-midline test of forelimb placing, the animal was gently held by its torso, but was turned sideways so that the vibrissae were perpendicular to the surface of the table. The downwardly oriented limb was gently restrained by the experimenter as the downwardly oriented vibrissae were brushed against a table edge once per trial for 10 trials. The percentage of trials in which the rat successfully places its other forepaw onto the tabletop was recorded for each side. Intact animals place the forelimbs of both sides quickly onto the counter top with $100 \%$ success in this test from P21. If an animal struggled during testing, the data were not included in the overall analysis. Damages to the nigrostriatal system led to a decrease in the successful rate of placing in the contralateral limb in this test. Due to the bilateral effect of rotenone, the percentage of successful placing response for both forelimbs was averaged together to create one score.

\section{Pole Test}

The rat was confronted with a situation in which it had to turn around and climb down a pole (diameter: $3 \mathrm{~cm}$, length: 50 $\mathrm{cm}$ ). The animal was placed under a cork ball installed at the top of the pole with its head held upwards. Each animal was given 3 trials weekly and the time to turn around and to reach the platform at the bottom was measured as performance latency (cutoff time: $60 \mathrm{~s}$ ). 
Tapered/Ledged Beam Walking Test

Rats were allowed to transverse a tapered beam with underhanging ledges on each side to permit foot faults without falling. The rats' performance was videotaped and later analyzed by calculating the slip ratio of the hind limb (number of slips/number of total steps). The time spent on the beam for each animal that traversed the beam was recorded. The slip ratios for both hind limbs were averaged to create one score. The mean of 3 trails was used for statistical analyses.

\section{Quantification of Data and Statistics}

Semi-quantification of OX42+ cell density at the SN was performed in 3 consecutive brain sections at the SN by an observer blinded to the treatment as described previously [18]. Three digital microscopic images were randomly captured in each of the $3 \mathrm{sec}$ tions and the number of positively stained cells in the 3 images was counted and averaged (cells $/ \mathrm{mm}^{2}$ ). The mean value of cell counting from the 3 brain sections was used to represent 1 single brain. For convenience of comparison among the treatment groups, results were standardized as the average number of cells $/ \mathrm{mm}^{2}$ in the $\mathrm{SN}$. In response to LPS challenge, the number of OX42+ microglia increases and the soma of these cells become larger. In addition to cell density, the OX42 immunoreactivity was also quantified using our previously developed method [18, 33], i.e., using computer software to determine the percentage area that contains OX42+ staining in the entire area of the captured image. This method has also been successfully used to quantify the OX42 staining in the SN $[18,19]$ and the density of cortical serotonin transporter-immunoreactive fiber networks [36].

Data from stereological cell counting, mitochondrial complex I activity, immunoblotting analysis, immunostaining, and ELISA were presented as the mean \pm SEM and analyzed by one-way ANOVA followed by the Student-Newman-Keuls test. The behavioral data were presented as the mean \pm SEM and analyzed by twoway repeated-measures ANOVA (for tests conducted continuously at different days), followed by the Student-Newman-Keuls test. Results with a $\mathrm{p}<0.05$ were considered statistically significant.

\section{Results}

\section{Peripherally Injected LPS Can Enter the Neonatal Brain and Result in Acute and Chronic Brain Inflammation}

Endogenous LPS concentration in the rat brain was very low, but it quickly increased to the range of 50-90 pg/mg protein in the rat brain 2-24 h after intraperitoneal injection of LPS (fig. 1), suggesting that peripherally administered LPS can reach the neonatal rat brain. Systemic exposure to LPS resulted in acute and chronic inflammation in the rat brain, as evidenced by the immediate and sustained elevation of inflammatory cytokine levels (fig. 2). Six hours following LPS injection ( $2 \mu \mathrm{g} / \mathrm{g}$, i.p.), TNF- $\alpha$ and IL- $1 \beta$ concentrations in the serum of LPS-exposed rats were dramatically increased $(2,789.8 \pm 245.7$ and $4,290.8 \pm 506.2 \mathrm{pg} /$ $\mathrm{ml}$, respectively) as compared to those in the saline-inject-

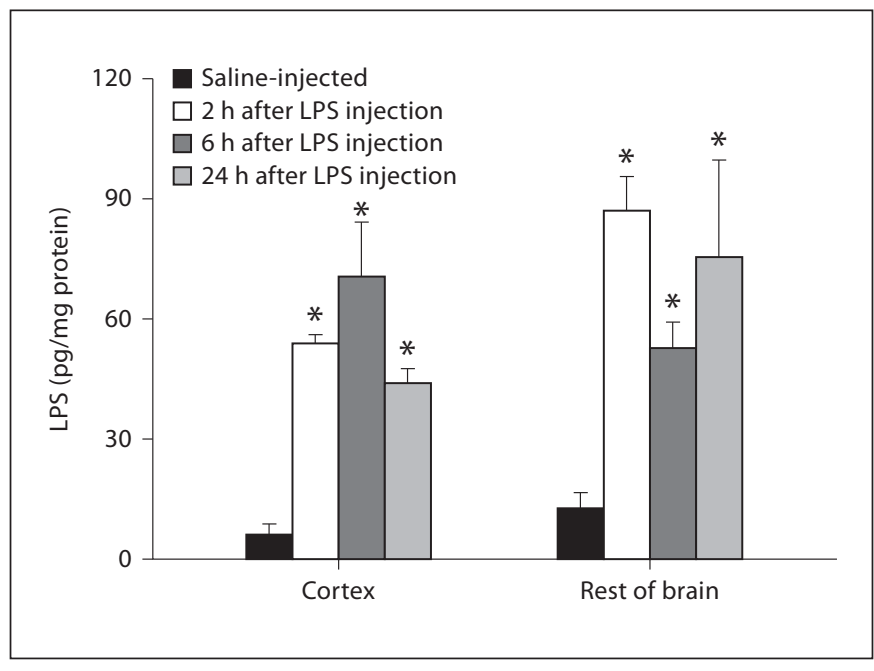

Fig. 1. Concentrations of LPS in the rat brain after systemic administration of LPS in P5 rats. LPS (from E. coli, serotype 055:B5) was intraperitoneally injected to P5 rats at a dose of $2 \mu \mathrm{g} / \mathrm{g}$ of body weight and the control rats were injected with the same volume of sterile saline. Concentrations of LPS in the rat brain were determined with an ELISA kit at designated time points. The results are expressed as the mean \pm SEM of 4 animals in each group, and analyzed by one-way ANOVA. ${ }^{*} \mathrm{p}<0.05$ different from the salineinjected group.

ed rats $(5.6 \pm 2.2$ and $2.1 \pm 0.7 \mathrm{pg} / \mathrm{ml}$, respectively). TNF- $\alpha$ and IL- $1 \beta$ concentrations in the LPS-exposed rat brain were also significantly increased $(10.6 \pm 0.2$ and $32.9 \pm 4.2$ $\mathrm{pg} / \mathrm{mg}$ protein, respectively) as compared to those in the control rat brain $(0.2 \pm 0.1$ and $4.8 \pm 0.9 \mathrm{pg} / \mathrm{mg}$ protein, respectively). As shown in figure $2 \mathrm{~A}-\mathrm{C}$, concentrations of TNF- $\alpha$, IL- $1 \beta$ and IL- 6 in the serum of LPS-exposed rats were still significantly higher than those in the control rats $24 \mathrm{~h}$ following LPS injection at a dose of $2-3 \mu \mathrm{g} / \mathrm{g}$ body weight. Regardless of the LPS dose, concentrations of TNF- $\alpha$ and IL- 6 in the rat brain returned to the control level $24 \mathrm{~h}$ after the LPS exposure. However, IL-1 $\beta$ concentration in the LPS-exposed rat brain remained elevated at $24 \mathrm{~h}$, when LPS was administered at a dose of $1-3 \mu \mathrm{g} / \mathrm{g}$ body weight. More impressively, concentrations of IL- $1 \beta$ in various regions of the LPS-exposed rat brain were persistently increased even at 65 days (P70) after the initial intraperitoneal injection (fig. 2D). Since LPS at a dose of $2 \mu \mathrm{g} / \mathrm{g}$ body weight was sufficient to induce chronic brain inflammation, this dose was selected in all further experiments.

Systemic LPS exposure-induced brain inflammation was also evidenced by the increased microglia activation, as indicated by the increased number of OX $42+$ cells and the increased OX42+ immunoreactive areas at $24 \mathrm{~h}$ (data 


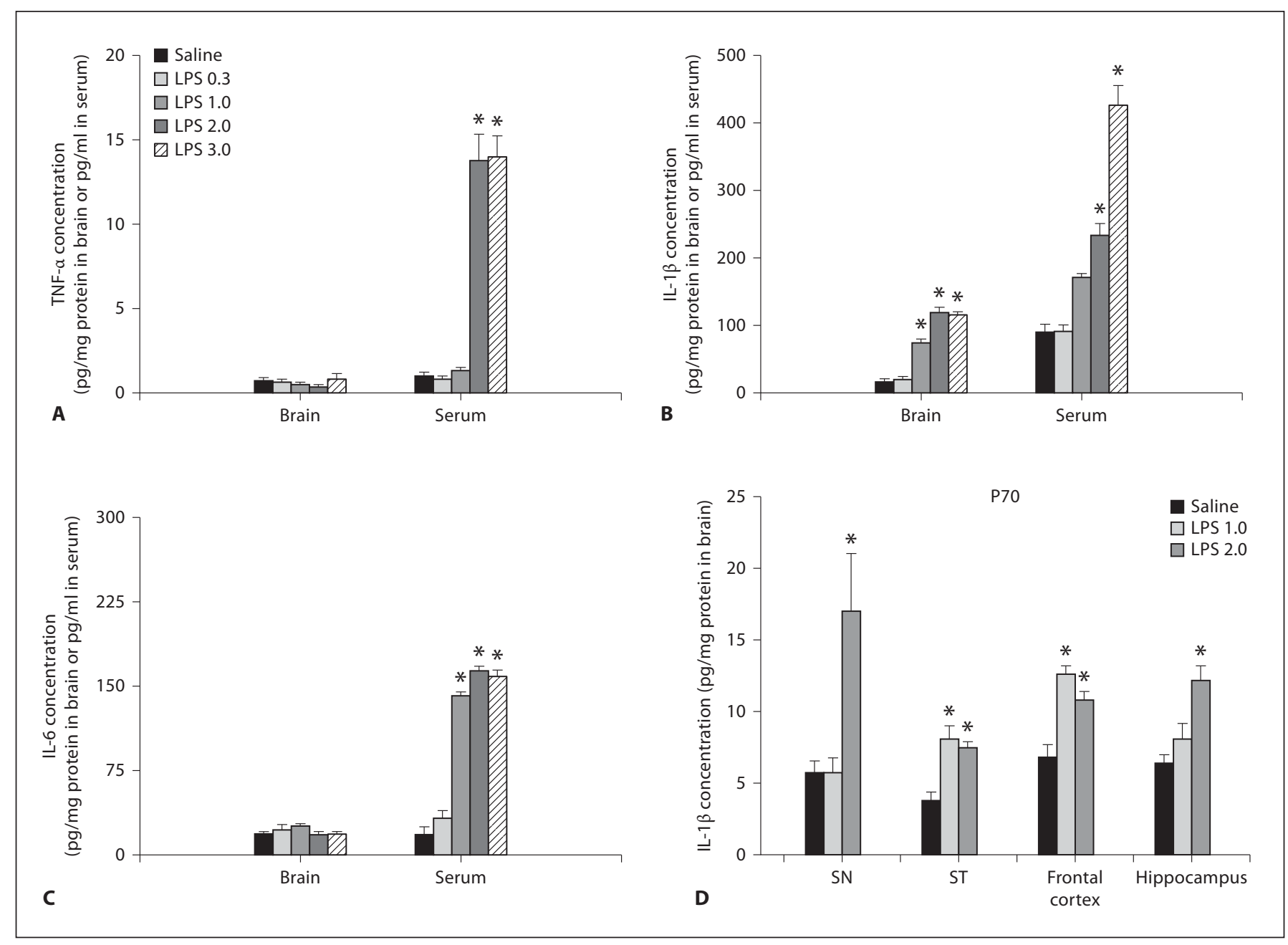

Fig. 2. Concentrations of inflammatory cytokines in the rat brain and serum $24 \mathrm{~h}$ (A-C) or 65 days (P70) (D) after the intraperitoneal injection with LPS at different doses in P5 rats. The control rats were injected with the same volume of sterile saline. Inflammatory cytokines were determined by ELISA kits. Twenty-four hours after LPS administration at a dose of 2 or $3 \mu \mathrm{g} / \mathrm{g}$ body weight, serum levels of TNF- $\alpha($ A), IL-1 $\beta$ (B) and IL-6 (C) were still elevated as compared with the saline-injected group. Regardless of LPS doses, concentrations of TNF- $\alpha(\mathbf{A})$ and IL-6 (C) in the rat brain

not shown) and at P70 (fig. 3). Most detected OX42+ cells in the control rat brain were at a resting status with a small rod-shaped soma and fine, ramified processes (fig. 3A). In contrast, numerous OX42+ cells in the SN of LPS-exposed P70 rat brain had an altered morphology, i.e. brightened staining with an enlarged cell body and blunt processes (fig. 3B). At P70, both OX42+ cell density and OX42+ immunostained area in the SN of LPS-exposed rat brain were significantly higher than in the control rat brain (fig. 3C). were not different from that in the control rat brain $24 \mathrm{~h}$ after LPS injection, but IL-1 $\beta$ (B) concentration in the LPS-exposed rat brain remained increased as compared with that in the control rat brain. Sixty-five days (P70) after intraperitoneal injection with LPS at 1 or $2 \mu \mathrm{g} / \mathrm{g}$ body weight, concentrations of IL- $1 \beta$ in a variety of brain regions remained significantly higher than that in the control rat brain (D). The results are expressed as the mean \pm SEM of 4 animals in each group, and analyzed by one-way ANOVA. ${ }^{*} \mathrm{p}<0.05$ different from the saline-injected group.

\section{Neonatal Systemic LPS Exposure Did Not Cause Actual Death of Dopaminergic Neuron, but Enhanced Vulnerability of Adult Rats to Neurotoxicity of Rotenone}

As shown in figure 4, neonatal systemic LPS exposure suppressed $\mathrm{TH}$ expression in the $\mathrm{SN}$ as indicated by the reduced number of $\mathrm{TH}+$ neurons in the $\mathrm{P} 70$ rat brain (fig. 4B, C). However, the total number of NeuN cells in the same area was not significantly different between the 

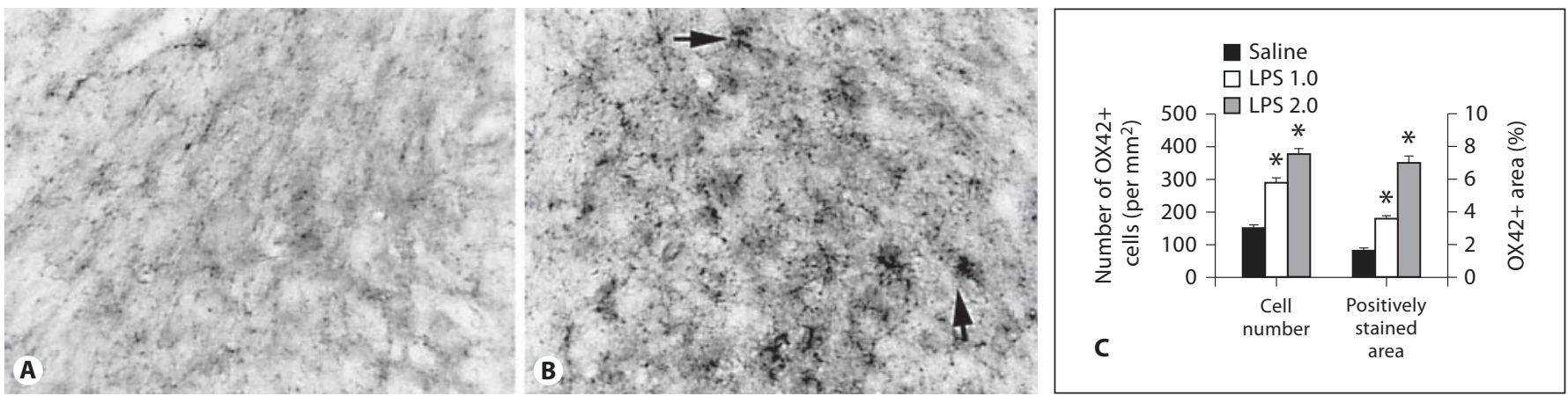

Fig. 3. Sustained microglial activation in the $P 70$ rat brain after the intraperitoneal injection of LPS on P5. Representative images of microglial immunostaining (OX42+) in the SN of P70 control or LPS-exposed rats are shown in A and B, respectively. Most microglia in the P70 control rat brain were weakly stained and at a resting status with a small rod-shaped soma and fine, ramified processes, while many OX $42+$ cells in the SN of LPS ( 1 or $2 \mu \mathrm{g} / \mathrm{g}$ body weight)-exposed P70 rat brain had bright staining of an enlarged cell body (arrows indicated) with blunt processes (B). Semi-quantification in terms of either OX42+ cell density or OX42+ stained area showed that microglia activation was persistently increased in the LPS-exposed rat brain $(\mathbf{C})$. The results in $\mathbf{C}$ are expressed as the mean \pm SEM of 5 animals in each group, and analyzed by one-way ANOVA. ${ }^{*} \mathrm{p}<0.05$ different from the saline-injected group.
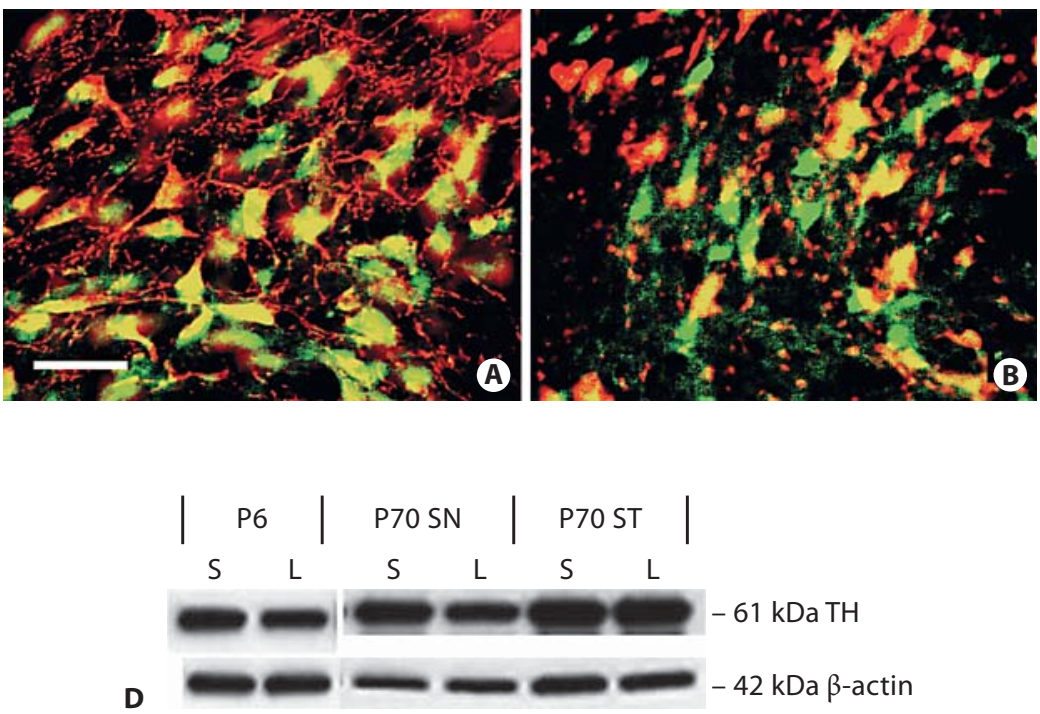

$61 \mathrm{kDa} \mathrm{TH}$

-42 kDa $\beta$-actin
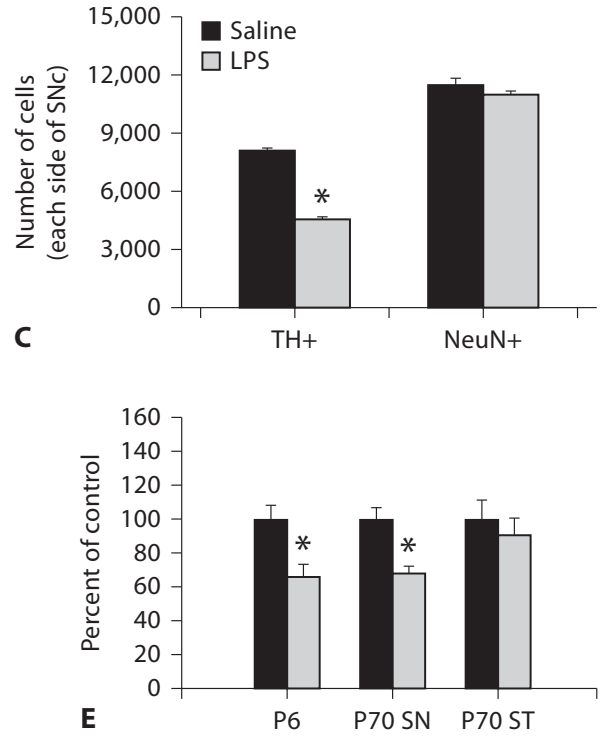

Fig. 4. Suppression of TH expression in neurons from the $\mathrm{SN}$ of P70 rats with neonatal LPS exposure. Representative merged photomicrographs of $\mathrm{TH}+$ (red) and $\mathrm{NeuN}+$ (green) cells in the SN of the rat brain from the control group and the LPS-exposed group are shown in $\mathbf{A}$ and $\mathbf{B}$, respectively (scale bar $=50 \mu \mathrm{m})$. Stereological cell counting showed that neonatal LPS exposure reduced number of $\mathrm{TH}+$ cells, but not $\mathrm{NeuN}+$ cells in the $\mathrm{SN}$ of $\mathrm{P} 70$ rats (C), suggesting that neonatal LPS exposure suppressed TH expres- sion, but did not cause significant neuronal death in the SN of P70 rat brain. Western blots for TH expression in the whole brain of P6 rats or in the SN or ST of P70 rats (D) and quantification of the blotting data (E) are consistent with immunohistochemistry results. The results in $\mathbf{C}$ and $\mathbf{E}$ are expressed as the mean \pm SEM of 5 animals in each group, and analyzed by t test. ${ }^{*} \mathrm{p}<0.05$ different from the saline-injected group. $\mathrm{SNc}=\mathrm{SN}$ pars compacta.
Early-Life Brain Inflammation and Risk for Neurodegeneration in Later Life
Dev Neurosci 2013;35:155-171 DOI: $10.1159 / 000346156$ 
LPS-exposed group and the control group (fig. 4A, C). Since the majority of neurons in the SN (85-90\%) are $\mathrm{TH}+$ dopaminergic neurons, the unaltered number of $\mathrm{NeuN}+$ cells indicated that neonatal LPS exposure may result in losses of TH immunoreactivity in dopaminergic neurons in the $\mathrm{SN}$ rather than actual cell death. The reduced TH expression in the LPS-exposed P6 rat brain as well as in the SN and ST of LPS-exposed P70 rat brain is shown with Western blots (fig. 4D, E), supporting the $\mathrm{TH}+$ cell counting data (fig. 4C).

After challenging with a small dose of rotenone, the total numbers of $\mathrm{TH}+$ and $\mathrm{NeuN}+$ cells in the $\mathrm{SN}$ were stereologically estimated in P98 rat brain. In the P98 saline + vehicle rat brain, $\mathrm{TH}+$ cells were more predominant in the SN (fig. 5E, M). Neonatal LPS exposure significantly reduced the number of $\mathrm{TH}+$ neurons in the $\mathrm{SN}$ of P98 rat brain (fig. 5G, O, Q). The rotenone treatment at a relatively low dose $(1.25 \mathrm{mg} / \mathrm{kg} /$ day for 14 days $)$ also slightly decreased the number of $\mathrm{TH}+$ neurons in the $\mathrm{SN}$ in the saline-exposed P98 brain (fig. 5F, N, Q). Neonatal LPS exposure enhanced the rotenone-induced decrease in the number of $\mathrm{TH}+$ neurons in the $\mathrm{SN}(\mathrm{p}<0.05)$ (fig. $5 \mathrm{H}, \mathrm{P}, \mathrm{Q}$ ) as compared to the LPS + vehicle group (fig. 5G, O).

As shown in the P70 LPS-exposed rat brain (fig. 4), neonatal LPS exposure did not significantly alter the total number of NeuN+ cells in the $\mathrm{SN}$ of $\mathrm{P} 98$ rat brain (fig. $5 \mathrm{~K}$, $\mathrm{O}, \mathrm{Q})$. However, rotenone treatment resulted in a significant decrease in the total number of $\mathrm{NeuN}+$ cells in the SN of P98 rat brain with neonatal LPS exposure $(\mathrm{p}<0.05)$ (fig. 5L, P, Q), but not in those without the neonatal LPS exposure (fig. 5J, N, Q). These data suggested that loss of TH immunoreactivity may represent a functional alteration while loss of $\mathrm{NeuN}+$ neurons may represent actual cell death in the SN.

To further address possible functional changes, as shown in figure 6, neonatal systemic LPS exposure-induced motor behavioral deficits, such as prolongation of the movement time [determined by the pole test (fig. $6 \mathrm{~B}$ ) and the tapered/ledged beam walking test (fig. 6C, D)] and prolongation of the reaction time [determined by the vibrissa-elicited forelimb-placing test (fig. 6A)], were spontaneously reversible, and by P70 all of the tested behaviors in the LPS-injected group reached the level of the control group. The rotenone treatment at P70 through subcutaneous minipump infusion at a relatively low dose of $1.25 \mathrm{mg} / \mathrm{kg} /$ day for 14 days resulted in neurobehavioral impairments in rats with the neonatal LPS exposure, but not in those without the neonatal LPS exposure (fig. 6).
Vibrissa-Elicited Forelimb-Placing Test

Regardless of neonatal exposure to LPS or vehicle, rats from all groups succeeded in the vibrissa-elicited forelimb-placing test (approx. 100\%) on P70 (fig. 6A). However, the success rate of the vibrissa-elicited forelimbplacing test in the LPS + rotenone group was significantly lower than that in the others from P77 to P98 ( $p<0.05)$ (fig. 6A).

\section{Pole Test}

On P70, rats from both saline- and LPS-exposed groups succeeded in the pole test (100\%) and the performance latency in the pole test was around 5-9 s (fig. 6B). Rotenone treatment produced a longer performance latency in the pole test in rats with the neonatal LPS exposure from P77 to P98 ( $<$ 0.05), but not in those without the neonatal LPS exposure (fig. 6B).

\section{Tapered/Ledged Beam Walking Test}

All P70 rats from either the saline- or the LPS-exposed group succeeded in the tapered/ledged beam walking test in less than $10 \mathrm{~s}$ with no foot faults (slips) made by the hind limbs, measured as an index of hind limb function (fig. 6C, D). The rotenone treatment significantly increased the number of hind limb foot faults in rats with the neonatal LPS exposure from P77 to P98 ( $\mathrm{p}<0.05)$, but not in those without the neonatal LPS exposure (fig. 6C). Following rotenone treatment, rats with the neonatal LPS exposure also needed longer time to complete the task (beam walking latency) from P77 to P98 ( $<<0.05$ ), as compared to those without the neonatal LPS exposure (fig. 6D).

\section{The Enhanced Vulnerability to Rotenone}

Neurotoxicity Was Associated with Persistent

Functional Alterations Induced by Neonatal Systemic

LPS Exposure

In investigating potential mechanisms involved in the enhanced vulnerability to rotenone neurotoxicity in adults with neonatal systemic LPS exposure, our data revealed that the enhanced vulnerability was associated with several persistent functional alterations following neonatal LPS exposure, in addition to the sustained elevation of IL-1 $\beta$ level in that rat brain (fig. 2D).

FG retrograde labeling pattern was used to examine dopaminergic nigrostriatal connectivity. Examples of photomicrographs of retrogradely labeled FG+ neurons in the SN of P70 rats with or without the neonatal LPS exposure are presented in figure $7 \mathrm{~A}, \mathrm{~B}$. In the control animals, numerous brightly FG-labeled nigrostriatal 

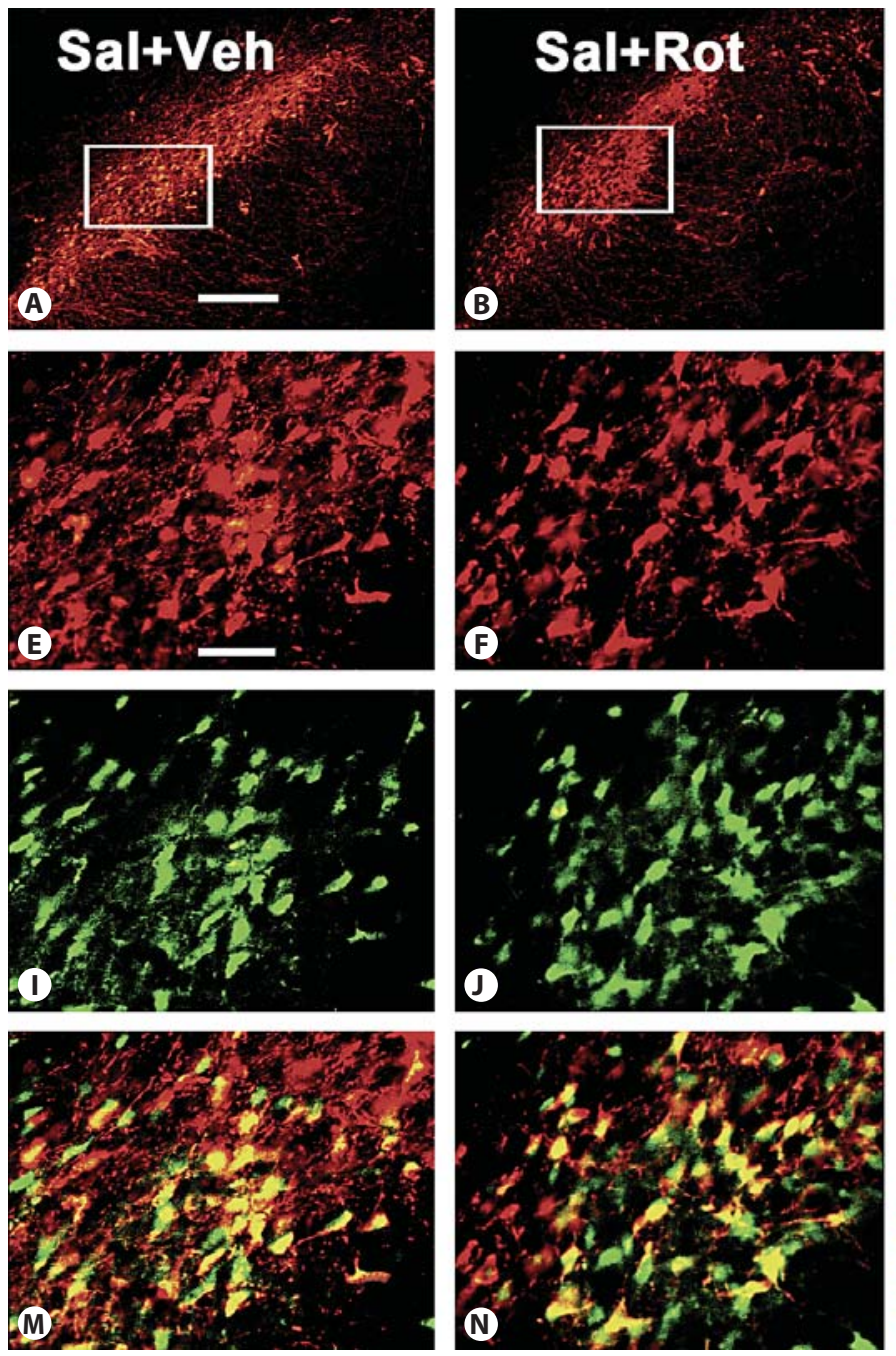

Fig. 5. Neonatal systemic LPS exposure enhanced rotenone effects on the number of $\mathrm{TH}+$ or NeuN+ cells in the SN of P98 rat brain. Representative photomicrographs of $\mathrm{TH}+$ cells (red) at low magnification (A-D) or high magnification (E-H), NeuN+ cells (green, I-L), and the merged images $(\mathbf{M}-\mathbf{P})$ in the $\mathrm{SN}$ of rat brain are shown here. Representative images for the saline + vehicle group are shown in $\mathbf{A}, \mathbf{E}$, I and $\mathbf{M}$; those for the saline + rotenone group in $\mathbf{B}, \mathbf{F}, \mathbf{J}$ and $\mathbf{N}$; those for the LPS + vehicle group in $\mathbf{C}, \mathbf{G}, \mathbf{K}$ and $\mathbf{O}$, and those for the LPS + rotenone groups in $\mathbf{D}, \mathbf{H}, \mathbf{L}$ and $\mathbf{P}$. Neonatal LPS alone reduced the number of TH+ cells $(\mathbf{G})$ in the SN of P98 rat brain, but did not alter the number of NeuN+ cells (K) in the same area. Rotenone treatment in adult life also slightly decreased the number of TH+ cells (F), but not the number of NeuN+ cells $(\mathbf{J})$ in the saline + rotenone rat brain. Rotenone treatment resulted in not only a further decreased number of $\mathrm{TH}+$ cells $(\mathbf{H})$, but also a significant loss of $\mathrm{NeuN}+$ cells $(\mathbf{L})$, indicating actual death of dopaminergic neurons in the SN. The scale bar shown in $\mathbf{A}$ (for $\mathbf{A}-\mathbf{D}$ ) represents $200 \mu \mathrm{m}$ and that in $\mathbf{E}$ (for all other images) $50 \mu \mathrm{m}$. Stereologic cell counting data of the number of TH+ cells and the number of NeuN+ cells in the $\mathrm{SN}$ area are shown in $\mathbf{Q}$. The results are expressed as the mean \pm SEM of 5 animals in each group, and analyzed by one-way ANOVA. ${ }^{*} \mathrm{p}<0.05$ different from the saline + vehicle group; ${ }^{* *} \mathrm{p}<0.05$ different from for the LPS + vehicle group. $\mathrm{SNc}=\mathrm{SN}$ pars compacta. For colors, see online versions.

Early-Life Brain Inflammation and Risk for Neurodegeneration in Later Life
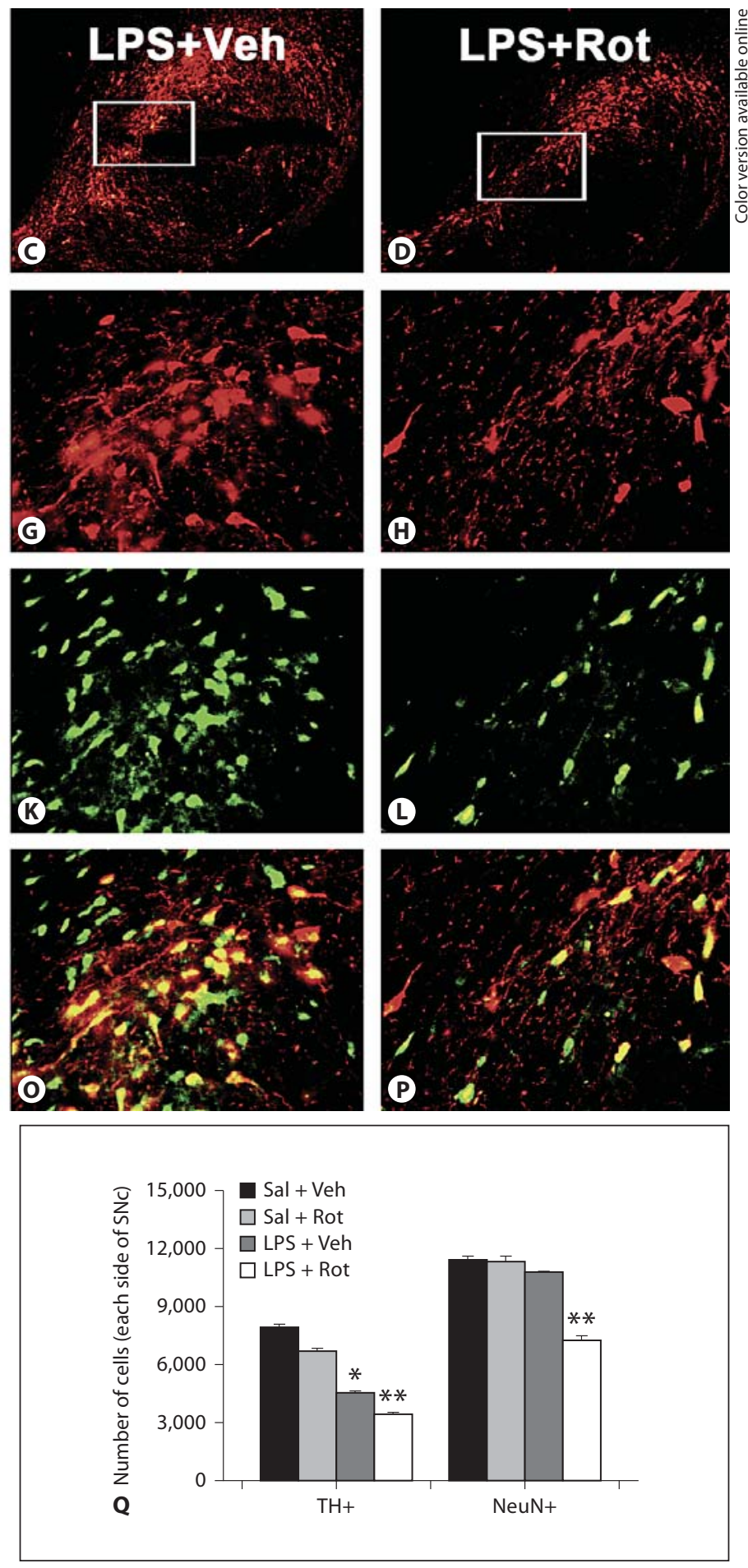

Dev Neurosci 2013;35:155-171 


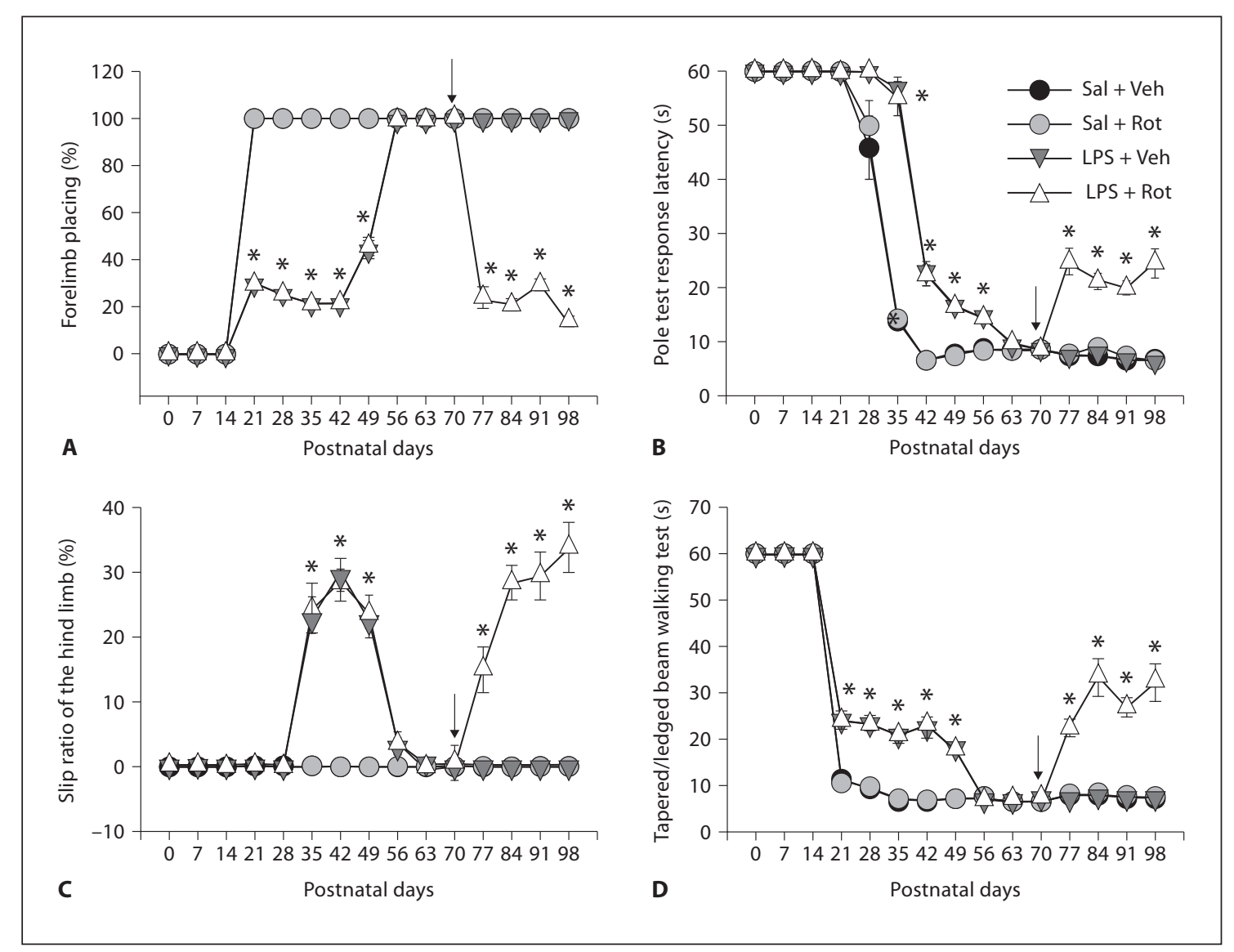

Fig. 6. Neurobehavioral tests in rats following neonatal systemic LPS exposure and rotenone infusion in adult life. A The vibrissaelicited forelimb-placing test. B The pole test. C Slip step ratio. D Performance latency in the tapered/ledged beam walking test. Neonatal LPS exposure resulted in motor dysfunction detectable in all the behavioral tests, but the motor deficits in LPS-exposed rats were recovered by P56-P70. Following rotenone challenge (indicated by an arrow in each panel), impaired motor performances were observed only in rats that had been exposed to LPS on P5, but not in other groups. The results are expressed as the mean \pm SEM of twelve animals in each group, and analyzed by twoway repeated measures ANOVA. ${ }^{*} \mathrm{p}<0.05$ different from all other groups on the same postnatal day. projection neurons with extensive dendritic processes were observed (fig. 7A). In contrast, less FG retrogradely labeled nigrostriatal projection neurons were observed in LPS-exposed animals and they tended to have fragmented dendritic processes (fig. 7B). These findings suggest that nigrostriatal dopaminergic neurons remain connected to the ST, but may not function properly and that the dopaminergic system in LPS-exposed rat brain was in a 'suboptimal' status. The nigral dopaminergic dendrites were further examined using an electron microscopy. Intriguingly, LPS-exposed nigral dendrites (labeled with letter $\mathrm{d}$ in fig. 7C, D) often exhibited rather sparse internal contents. Those disintegrated dendrites contain numerous vacuoles (arrow indicated in fig. 7D) and even myelinated axon inclusions (marked with an asterisk), as well as lack of mitochondria (fig. 7D). Normal dendrites with internal structures such as mitochondria (as indicated by an arrowhead in fig. 7C) and synaptic contacts (pointed by white arrows) are noted in saline-treated control animals (fig. 7C). Interestingly, these alterations in the dopaminergic system were closely associated with lasting changes in mitochondria complex I activity. Following LPS exposure at a dose of $2 \mu \mathrm{g} / \mathrm{g}$ (i.p.) on P5, mitochondria complex I activity was significantly decreased in the P6 whole rat brain and the SN of P70 rats, respectively (fig. 7E, F).

The 'suboptimal' dopaminergic system following neonatal systemic LPS exposure was also reflected by the al- 
Fig. 7. Neonatal systemic LPS exposure resulted in long-lasting damage to dopaminergic neuronal connectivity determined by FG retrograde labeling in the rat brain and to mitochondrial function. FG retrogradely labeled nigrostriatal dopaminergic neurons in control rats were rather bright and containing extensive smooth dendritic processes $(\mathbf{A})$. In contrast, the number of FG-labeled dopaminergic neurons in LPSexposed animals tended to be less and fragmented dendritic processes were observed (B). Ultrastructural characteristics of neuroprofiles in the $\mathrm{SN}$ of P70 control and LPSexposed rat brain are shown in $\mathbf{C}$ and $\mathbf{D}$, respectively. Dendrites (labeled with letter d in $\mathbf{C}$ and $\mathbf{D}$ ) with internal structures such as mitochondria (as indicated by an arrowhead in $\mathbf{C}$ ) and synaptic contacts (pointed by white arrows in $\mathbf{C}$ ) were noted in salinetreated control animals (C). Dendrites in the LPS-exposed rats often had sparse internal contents and contained many vacuoles (arrow indicated in D) and even myelinated axon inclusions (marked with an asterisk), and lacked mitochondria and lost synaptic contacts. Neonatal LPS exposure significantly reduced mitochondrial complex I activity (nmol NADH-ubiquinone oxidoreductase/min/mg protein) in the P6 rat brain (E) and the SN of P70 rat brain (F). The scale bar in $\mathbf{A}$ (for $\mathbf{A}$ and $\mathbf{B}$ ) represents 50 $\mu \mathrm{m}$, and in $\mathbf{C}$ (for $\mathbf{C}$ and $\mathbf{D}$ ) it represents 500 $\mathrm{nm}$. The results in $\mathbf{E}$ and $\mathbf{F}$ are expressed as the mean \pm SEM of 5 animals in each group, and analyzed by one-way ANOVA. * $\mathrm{p}<$ 0.05 different from the saline group.
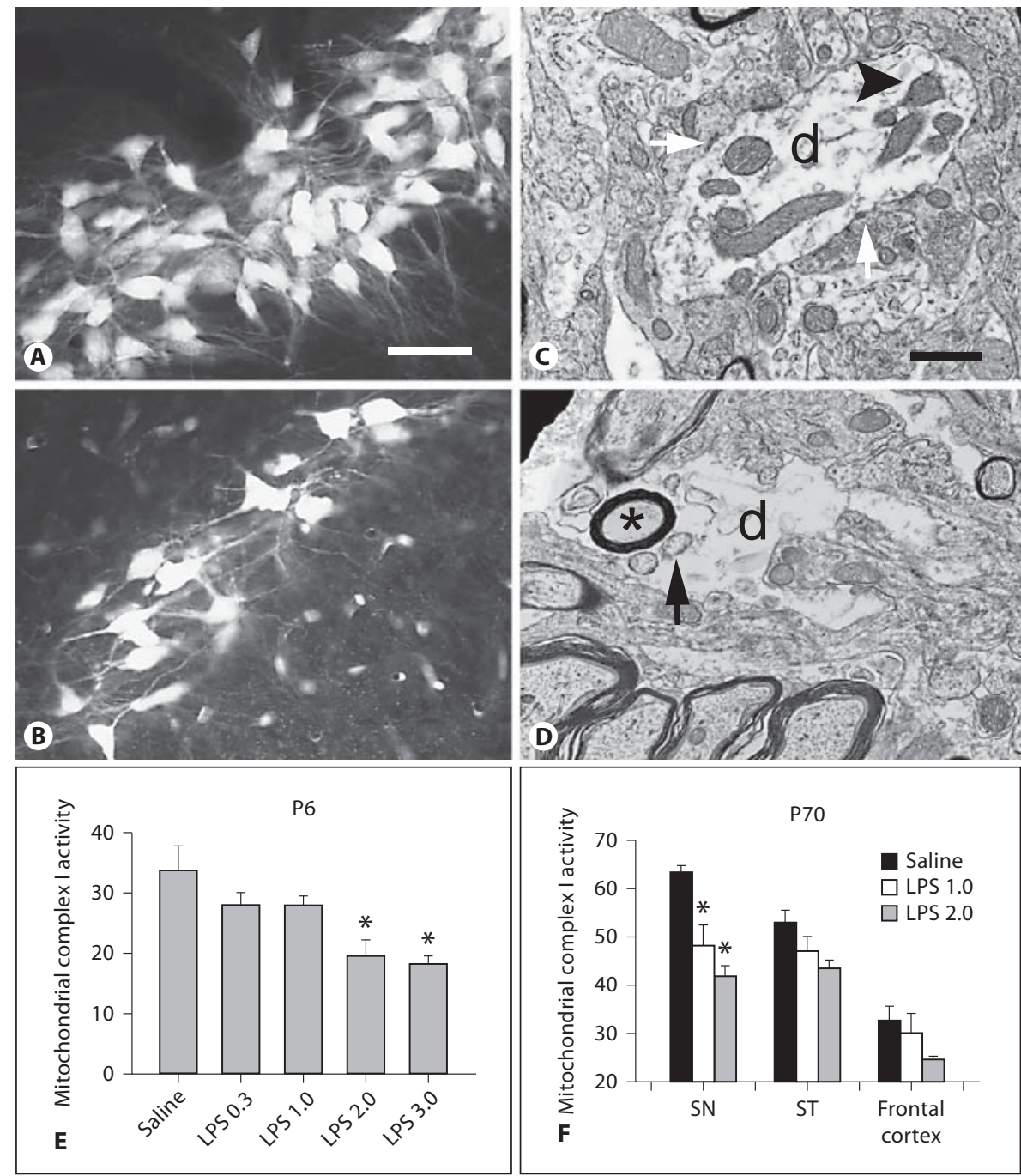

tered expression of COX-2 and p-p38 MAPK in the P70 rat brain (fig. 8). Increased expression of COX-2, the inducible cyclooxygenase, in the brain is thought to play important roles in PD neurodegeneration [37]. Neonatal systemic LPS exposure resulted in sustained inflammatory responses, as indicated by the increase in the number of COX-2+ cells in the SN of P70 rat brain (fig. 8B, C) as compared to that in the control rat brain (fig. 8A, C). Double-labeling data showed that most COX-2+ cells in the $\mathrm{SN}$ were $\mathrm{NeuN}+$ neurons (fig. $8 \mathrm{~B}$ ), but very few of them were $\mathrm{TH}+$ neurons (data not shown). In addition, a few COX-2+ cells were also OX42-expressing microglia, but no astrocytes were found to express COX-2 (data not shown). As a downstream event of IL-1 signaling, p38 MAPK plays important roles in dopaminergic neuron death in animal models of PD [38]. In the current study, p-p38 MAPK, but not p38 MAPK expression was significantly increased in P6 and the SN and ST of P70 LPSexposed rat brain (fig. 8D, E), suggesting that p38 MAPK in the SN may function abnormally following neonatal LPS exposure.

The enhanced vulnerability to rotenone neurotoxicity in LPS-exposed rats was also found to be linked with an enhanced activation of microglia triggered by a small dose of rotenone. Similar to the P70 data shown in figure 3 , most detected OX42+ cells in the SN of P98 control rat brain were at a resting stage with a small rod-shaped soma and fine, ramified processes (fig. 9A, G), while neonatal LPS exposure resulted in a sustained increase in microglial activation in the P98 rat brain, as indicated by the increased OX42+ cell density (fig. 9B, E) or the increased OX42+ staining area (fig. 9F), as well as altered morphol- 

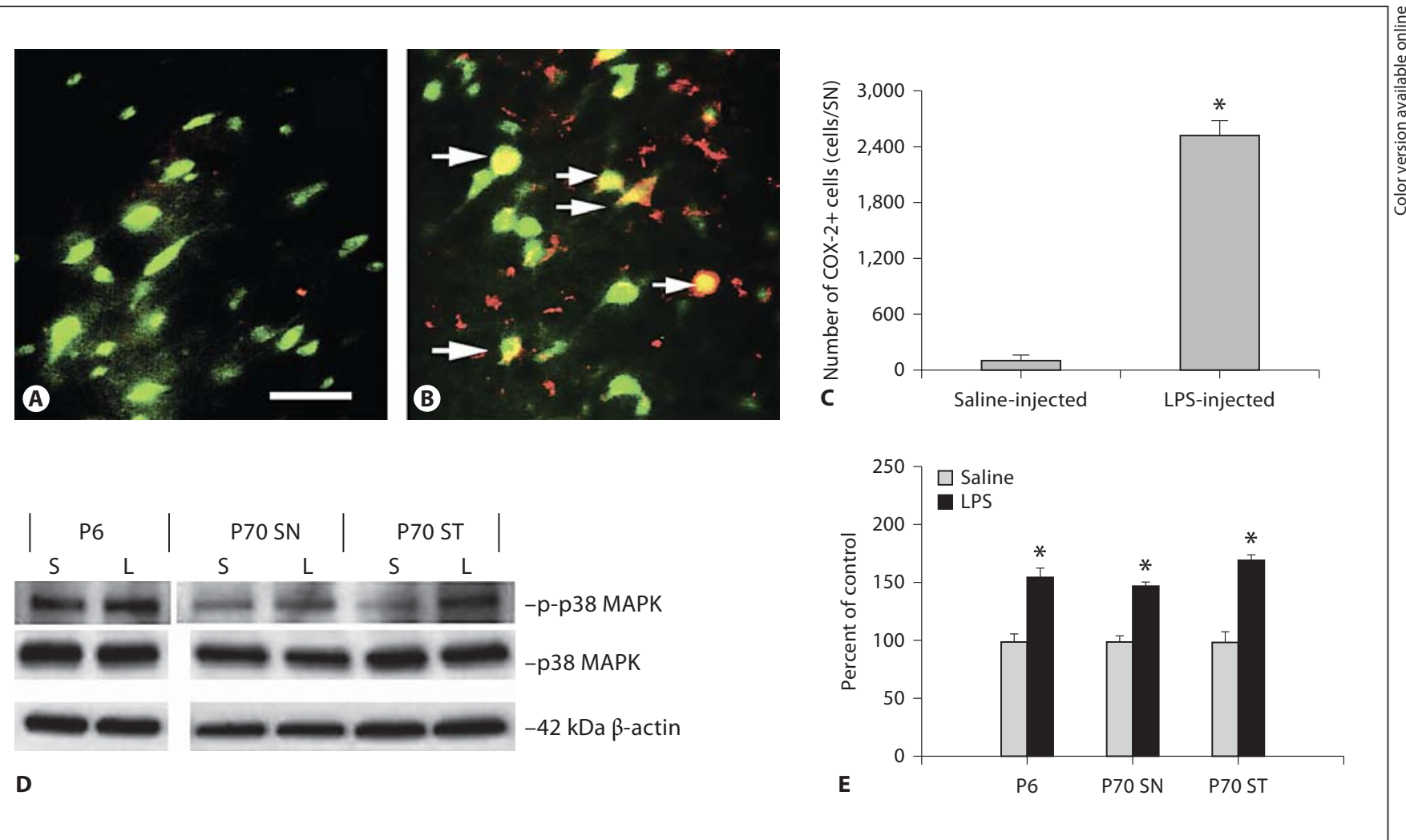

Fig. 8. Neonatal LPS-exposed persistently increased COX-2 expression and phosphorylation of p38 MAPK in the P70 rat brain. Representative photomicrographs of COX-2+ (red) and NeuN+ (green) cells in the SN of P70 control rat brain and LPS-exposed rat brain are shown in $\mathbf{A}$ and $\mathbf{B}$ (scale bar $=50 \mu \mathrm{m}$ ). Increased COX$2+$ cells were observed in the SN of LPS-exposed rat brain, while almost no COX-2+ cells were found in the control rat brain $(\mathbf{A})$. Many COX-2+ cells found in the LPS-exposed rat brain were

ogy of the OX42+ cells. For example, numerous OX42+ cells in the SN of LPS-exposed rat brain showed typical features of activated microglia, i.e. bright staining with an elongated cell body and blunt processes (fig. 9H) [39]. With rotenone exposure only, increased OX42+ cell density and staining area in the $\mathrm{SN}$ of the saline + rotenone group was noted as compared to the control (fig. 9C, E, F; $\mathrm{p}<0.05)$. Interestingly, the morphology of these OX $42+$ cells was similar to those in the LPS + vehicle group (fig. $9 \mathrm{H}$ ). On the contrary, the same dose of rotenone triggered a much stronger response of microglia in the SN with neonatal LPS exposure, suggesting further inflammatory responses. The density of OX42+ cells or the OX42+ staining area in the SN of the LPS + rotenone group was further significantly increased as compared to the LPS + vehicle group (fig. 9D-F; p < 0.05). Under such
NeuN+ neurons (arrows indicated in B). Stereological cell counting data of COX-2+ cells are shown in C. Western blot analysis (D) showed that neonatal LPS significantly increased p38 phosphorylation in the P6 rat brain and the SN and ST of P70 rat brain. Semiquantification of the blotting data is shown in $\mathbf{E}$. The results in $\mathbf{C}$ and $\mathbf{E}$ are expressed as the mean \pm SEM of 5 animals in each group, and analyzed by $\mathrm{t}$ test. ${ }^{*} \mathrm{p}<0.05$ different from the saline group. For colors, see online version. treatments, the soma of OX42+ cells was further enlarged (fig. 9I) and exhibited a rather round-shaped body with minimum processes (fig. 9J).

\section{Discussion}

The major finding of the present study is that systemically administered LPS can penetrate into the neonatal rat brain and cause acute and chronic brain inflammation. Our results have further demonstrated that as does LPS exposure through intracerebral injection [19], although neonatal systemic LPS exposure may not necessarily lead to death of dopaminergic neurons in the $\mathrm{SN}$, such an exposure could cause persistent functional alterations in the dopaminergic system and indirectly predis- 

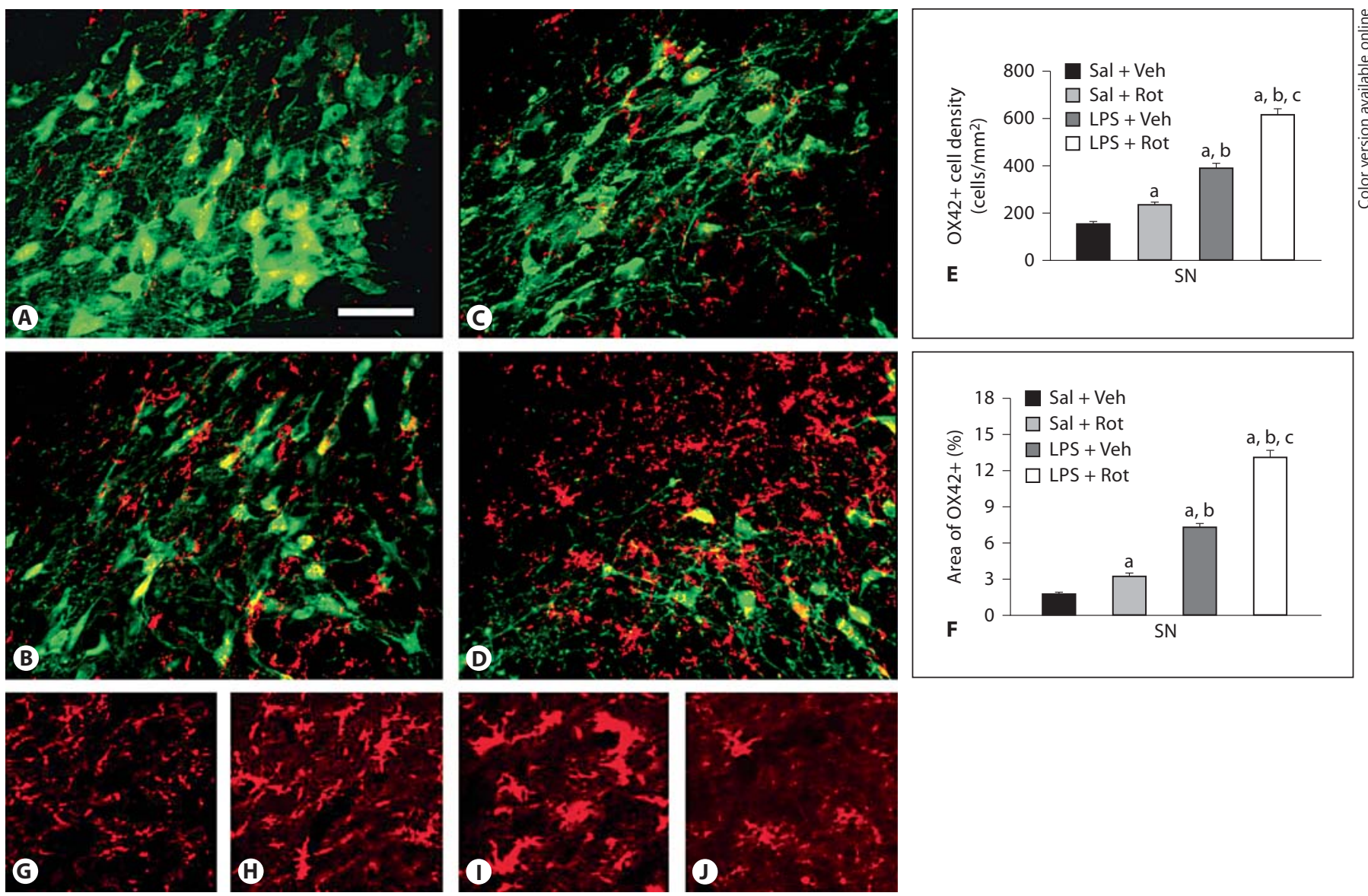

Fig. 9. Neonatal LPS exposure enhanced microglia activation triggered by a small dose of rotenone in the P98 rat brain. Representative photomicrographs of OX42 (red) and NeuN (green) immunostaining in the SN are shown for the saline + vehicle group (A); the LPS + vehicle group (B); the saline + rotenone group $(\mathbf{C})$, and the LPS + rotenone group (D). The quantitative data of microglia activation are presented as the density of OX42+ cells in the SN (E) or the percentage area that contains OX42+ staining in the images (F). Microglia at a resting status with a small rod-shaped soma and ramified processes $(\mathbf{G})$ were detected in the $\mathrm{SN}$ of the saline + vehicle group (A). Neonatal LPS exposure resulted in chronic activation of microglia in the SN of the LPS + vehicle group, as indicated by the increased density of OX $42+$ cells (E) or increased percentage area containing OX42 staining (F) and the altered cell morphology (elongated cell body with blunt processes, as shown in $\mathbf{H}$ ). Rotenone treatment at adult life also activated microglia in the SN of the saline + rotenone group $(\mathbf{C})$, as indicated by the increased density of OX $42+$ cells (E) and OX42+ stained area (F). But morphology of OX42+ cells in this group remained similar to those in LPS + vehicle group. Activation of microglia triggered by this dose of rotenone was enhanced in the LPS + rotenone group (D), as indicated by the further increased density of OX42+ cells (E) or further increased percentage area containing OX42 staining (F) as compared to that in the LPS + vehicle group. The soma of OX42+ cells in this group was further enlarged and many of them lost processes (I) or even became round-shaped (J). The scale bar in $\mathbf{A}$ represents $50 \mu \mathrm{m}$. The results in $\mathbf{E}$ and $\mathbf{F}$ are expressed as the mean \pm SEM of 5 animals in each group, and analyzed by one-way ANOVA. ${ }^{a} \mathrm{p}<0.05$ different from the saline + vehicle group; ${ }^{b} \mathrm{p}<0.05$ different from the saline + rotenone group, and ${ }^{\mathrm{c}} \mathrm{p}<0.05$ different from the LPS + vehicle group. pose the nigrostriatal system in the adult brain to be damaged by environmental toxins at an ordinarily nontoxic or subtoxic dose and to develop PD-like pathological features and motor dysfunction.

Perinatal or early-life exposure to an endotoxin, LPS, has been shown to increase the risk for dopaminergic dis- orders in animal models of PD $[9,10]$. However, the question of how perinatal LPS exposure could lead to the development of neurodegenerative diseases such as PD in late life remains to be answered. Prenatal exposure (E10.5) to LPS in rats reduces the number of dopaminergic neurons in the $\mathrm{SN}$ of adult brain, indicating that prenatal cen- 
tral nervous system inflammation, such as that which is caused by exposure to LPS, may contribute to the development of PD later in life [10]. Following prenatal LPS exposure, however, progressive dopaminergic neuron loss, as observed in human patients with PD, requires a second dose of LPS challenge in adult rats [11]. Data from our previous $[17,19]$ and current studies suggest that neonatal exposure to LPS in P5 rat brain results in lesions in the nigrostriatal dopaminergic system and functional injury, but does not cause actual dopaminergic neuron death. The different exposure time (i.e., pre- vs. postnatal) may explain such differences between our study and that reported by Ling et al. [10]. The rat dopaminergic system develops both pre- and postnatally, with dopaminergic neuron birth, specification and migration to the final position occurring prenatally and its receptor development and the establishment of contact between the SN and other neural nuclei largely occurring postnatally [40, 41]. It is likely that LPS exposure on E10.5, when dopaminergic neurons are born [40], might impair their neurogenesis and thus reduce their numbers, while either systemic or central [19] LPS exposure on P5, when birth of dopaminergic neurons is already completed, may result in partial degeneration rather than true neuron death. Results from the present study are consistent with the synergic effects between LPS and rotenone reported by Ling et al. [26] that rotenone potentiates dopaminergic neuron loss in animals exposed to LPS prenatally. However, in that reported study, prenatal LPS already results in dopaminergic neuron death and rotenone is considered to enhance the LPS-induced neuron death. There might be different mechanisms involved in the synergic effects between LPS and rotenone reported in this study as compared to that reported by Ling et al. [26].

Data from the present study indicate that even in the presence of dopaminergic system partial degeneration in the adult rat brain that has been systemically exposed to LPS on P5, along with development, the neonatal LPS exposure-induced motor function deficits could spontaneously recover by P70 (fig. 6), while the dopaminergic system in these rat brains was more susceptible to be further injured. These results suggest that neonatal systemic LPS exposure may result in a state of silent neurotoxicity in the adult brain $[42,43]$, rather than neuronal death. Although it is unknown if the observed neuroinflammation and mitochondrial complex I inhibition in the LPS-exposed P70 rat brain might be sufficient to cause dopaminergic neurodegeneration in very aged rats, data from the present study indicate that the pre-existing brain injuries may not be severe enough to cause apparent neurological deficits or could be functionally compensated, but they may lead to much severer damages upon further stimuli, which are ordinarily not harmful. In the current study, P70 rats, in which neonatal systemic LPS exposure-induced motor deficits were already recovered, were challenged with a relatively low dose of rotenone $(1.25 \mathrm{mg} / \mathrm{kg} /$ day, 14 days). Severe damages to the dopaminergic system such as actual loss of dopaminergic neurons (fig. 5) and PD-like neurobehavioral dysfunctions (fig. 6) were observed only in those exposed to LPS on P5, but not in those without the neonatal LPS exposure. Our findings strongly support the notion that a silent neurotoxic state induced by neonatal brain inflammation may enhance susceptibility in adults to the development of neurodegenerative disorders, such as PD, triggered by environmental toxins at an ordinarily nontoxic or subtoxic dose. Perinatal infection and inflammation occurs frequently in both preterm and full-term infants. Increasing evidence indicates that neonatal brain injury is commonly associated with infection/inflammation $[1,44]$. If findings from the current study are proven to be valid in humans, the silent neurotoxic state may exist in many survivors with perinatal brain injury following systemic infection/inflammation. Such a population may be at a high risk for development of neurodegenerative disorders in their late life when they are exposed to environmental toxins, such as pesticides and herbicides, which may be commonly present at a very minimum level in our daily life and are not considered harmful to ordinary individuals. Thus, whether or not the potential silent neurotoxic state exists in the human population, its prevention is certainly worth further investigation.

Although factors involved in the enhanced vulnerability of the dopaminergic system in the rat brain with a neonatal LPS exposure remain unclear, several sustained functional alterations in the adult rat brain with neonatal systemic LPS exposure might contribute to the enhanced vulnerability of the dopaminergic system. The essential mechanism of rotenone neurotoxicity is its inhibitory effects on the mitochondrial respiratory chain [45]. Our previous studies show that mitochondrial complex I activity in the SN of P70 rat brain with neonatal central LPS exposure was significantly reduced as compared to that in the control group [18] and a challenge with an ordinarily subtoxic dose of rotenone resulted in dopaminergic neuron death in the $\mathrm{SN}$ along with significant further decreases in mitochondrial complex I activity [19]. Chronic inhibition of the mitochondrial complex I has been shown to selectively damage dopaminergic neurons despite being uniformly distributed throughout the brain [46]. There- 
fore, persistently compromised mitochondrial function in the SN following neonatal systemic LPS exposure in our study could contribute to the enhanced vulnerability of the dopaminergic systems in this animal model.

Expression of COX-2 has been shown to be specifically induced in dopaminergic neurons in the SN in postmortem PD subjects and in the MPTP mouse model of PD [47]. Increased susceptibility to excitotoxicity in COX-2-overexpressing neurons and neuroprotection by COX-2 inhibition has been shown in several experimental PD models [48]. Therefore, increased expression of COX-2 in the brain is considered to play important roles in PD neurodegeneration [37]. In the present study, persistently increased expression of COX-2 in the SN neurons was found in P70 rat brain following neonatal LPS exposure (fig. 8) and thus, it could also be a potential contributor to the enhanced vulnerability of the dopaminergic systems in this animal model.

Chronic activation of microglia in the $\mathrm{SN}$ following neonatal LPS could be another contributor to the enhanced vulnerability of the dopaminergic systems. Postmortem analyses $[49,50]$ and in vivo imaging studies using PET ligands [51] have shown microglial activation in the SN of patients with PD. Activation of microglia is known to play an important role in the initiation and progression of PD $[45,52,53]$. Microglial activation involves not only increases in the number of microglia, but also the elevation of inflammatory molecules released from the activated microglia, such as cytokines, chemokines, reactive oxygen and nitrogen species $[53,54]$, which can directly lead to the dysfunction and degeneration of dopaminergic neurons [55]. The current study indicates that chronically activated microglia alone in the LPS-exposed P70 rat is not associated with actual dopaminergic neuron death and the associated neurological deficits, but it did link with a sustained elevation of IL- $1 \beta$ and phosphorylation of p38 MAPK, a downstream event of IL-1 signaling which is known to play important roles in the death of dopaminergic neurons in animal models of PD [38] (fig. 2, 8). It appears that the effects of chronically activated microglia and the additional outcomes triggered by a small dose of rotenone are synergetic or additive. Neonatal LPS-induced chronic activation of microglia alone may not trigger severe enough adverse effects on the dopaminergic system, but neonatal LPS exposure may prime the microglia [56], and upon the rotenone further challenge at late life, the primed microglia may then produce severe enough responses to induce actual dopaminergic neuron loss and PD-like neurological deficits, as we reported here and previously [19]. An interesting finding in the present study is the morphology of many chronically activated microglia in LPS-exposed rat brain, which have an elongated cell body with shorter processes (fig. 9H) as compared to those in the control rat brain (fig. 9G). Recent studies have shown that microglia can be activated to different stages according to their morphology, expressed markers, and secreted products $[39,57]$, and that activation of microglia may have dual effects following brain injury, either detrimental or beneficial [58]. Only microglia at certain stages may play critical roles in the degeneration of dopaminergic neurons [39]. In the present study, whether the shape of chronically activated microglia represents a specific activation stage or a feature of primed microglia as well as exact roles of these microglia in the enhanced vulnerability of the dopaminergic system are worth further investigations.

In brief, the persistent functional or structure alterations following neonatal systemic LPS exposure may individually or collectively contribute to the enhancement of susceptibility of adult brain to development of neurodegenerative disorders upon challenge with environmental toxins at a normally nontoxic dose, but further investigations regarding biological mechanisms are warranted. Although results from the present study are introductive, rather than conclusive, our animal model has provided an opportunity to explore the potential relationship between early-life brain inflammation and the occurrence of neurodegenerative diseases in later life. Such investigations may help us to better understand some mechanisms involved in the pathogenesis of nonfamilial PD and other neurodegenerative diseases.

\section{Acknowledgement}

This work was supported by a R01 research grant from the NIH (NS 54278).

References

\footnotetext{
1 Hagberg H, Gressens P, Mallard C: Inflammation during fetal and neonatal life: implications for neurologic and neuropsychiatric disease in children and adults. Ann Neurol 2012; 71:444-457.

2 Meyer U, Yee BK, Feldon J: The neurodevelopmental impact of prenatal infections at different times of pregnancy: the earlier the worse? Neuroscientist 2007;13:241-256.

-3 Meyer U, Feldon J, Fatemi SH: In-vivo rodent models for the experimental investigation of prenatal immune activation effects in neurodevelopmental brain disorders. Neurosci Biobehav Rev 2009;33:1061-1079.
}

Early-Life Brain Inflammation and Risk for Neurodegeneration in Later Life 
4 Patterson PH: Neuroscience. Maternal effects on schizophrenia risk. Science, 2007;318:576577.

5 Ghiani CA, Mattan NS, Nobuta H, Malvar JS, Boles J, Ross MG, Waschek JA, Carpenter EM, Fisher RS, de Vellis J: Early effects of lipopolysaccharide-induced inflammation on foetal brain development in rats. ASN Neuro 2011;3:pii,e00068.

6 Abdeslam M: Prenatal immune stress in rats dampens fever during adulthood. Dev Neurosci 2012;34:318-326.

7 Liu B, Gao HM, Hong JS: Parkinson's disease and exposure to infectious agents and pesticides and the occurrence of brain injuries: role of neuroinflammation. Environ Health Perspect 2003;111:1065-1073.

8 Logroscino G: The role of early life environmental risk factors in Parkinson's disease: what is the evidence? Environ Health Perspect 2005; 113:267-275.

-9 Feleder C, Tseng KY, Calhoon GG, O’Donnell $P$ : Neonatal intrahippocampal immune challenge alters dopamine modulation of prefrontal cortical interneurons in adult rats. Biol Psychiatry 2010;67:386-392.

$>10$ Ling Z, Gayle DA, Ma SY, Lipton JW, Tong CW, Hong JS, Carvey PM: In utero bacterial endotoxin exposure causes loss of tyrosine hydroxylase neurons in the postnatal rat midbrain. Mov Disord 2002;17:116-124.

-11 Ling Z, Zhu Y, Tong C, Snyder JA, Lipton JW, Carvey PM: Progressive dopamine neuron loss following supra-nigral lipopolysaccharide (LPS) infusion into rats exposed to LPS prenatally. Exp Neurol 2006;199:499-512.

-12 Carvey PM, Chang Q, Lipton JW, Ling Z: Prenatal exposure to the bacteriotoxin lipopolysaccharide leads to long-term losses of dopamine neurons in offspring: a potential, new model of Parkinson's disease. Front Biosci 2003;8:s826-s837.

13 Ling Z, Zhu Y, Tong C, Snyder JA, Lipton JW, Carvey PM: Prenatal lipopolysaccharide does not accelerate progressive dopamine neuron loss in the rat as a result of normal aging. Exp Neurol 2009;216:312-320.

14 Landrigan PJ, Sonawane B, Butler RN, Transande L, Callan R, Droller D: Early environmental origins of neurodegenerative disease in later life. Environ Health Perspect 2005; 113:1230-1233.

15 Miller DB, O'Callaghan JP: Do early-life insults contribute to the late-life development of Parkinson and Alzheimer diseases? Metabolism 2008;57(suppl 2):S44-S49.

16 Fan LW, Michell HJ, Rhodes PG, Cai Z: Alpha-phenyl-n-tert-butyl-nitrone attenuates lipopolysaccharide-induced neuronal injury in the neonatal rat brain. Neuroscience 2008; 151:737-744.

17 Fan LW, Tien LT, Mitchell HJ, Rhodes PG, Cai Z: $\alpha$-Phenyl-n-tert-butyl-nitrone ameliorates hippocampal injury and improves learning and memory in juvenile rats following neonatal exposure to lipopolysaccharide. Eur J Neurosci 2008;27:1475-1484.
18 Fan LW, Tien LT, Zheng B, Pang Y, Lin RCS, Simpson KL, Ma T, Rhodes PG, Cai Z: Dopaminergic neuronal injury in the adult rat brain following neonatal exposure to lipopolysaccharide and the silent neurotoxicity. Brain Behav Immun 2011;25:286-297.

19 Fan LW, Tien LT, Lin RC, Simpson KL, Rhodes PG, Cai Z: Neonatal exposure to lipopolysaccharide enhances vulnerability of nigrostriatal dopaminergic neurons to rotenone neurotoxicity in later life. Neurobiol Dis 2011; 44:304-316.

20 Betarbet R, Sherer TB, MacKenzie G, GarciaOsuna M, Panov AV, Greenamyre JT: Chronic systemic pesticide exposure reproduces features of Parkinson's disease. Nat Neurosci 2000;3:1301-1306.

21 Fleming SM, Zhu C, Fernagut PO, Mehta A, Dicarlo CD, Seaman RL, Xhesselet MF: Behavioral and immunohistochemical effects of chronic intravenous and subcutaneous infusions of varying doses of rotenone. Exp Neurol 2004; 187:418-429.

22 Gao HM, Hong JS, Zhang W, Liu B: Synergistic dopaminergic neurotoxicity of the pesticide rotenone and inflammogen lipopolysaccharide: relevance to the etiology of Parkinson's disease. J Neurosci 2003;23:1228-1236.

23 Sherer TB, Kim JH, Betarbet R, Greenamyre JT: Subcutaneous rotenone exposure causes highly selective dopaminergic degeneration and alpha-synuclein aggregation. Exp Neurol 2003;179:9-16.

24 Sulzer D: Multiple hit hypotheses for dopamine neuron loss in Parkinson's disease. Trends Neurosci 2007;30:244-250.

25 Simpson K, Wang Y, Lin RC: Patterns of convergence in rat zona incerta from the trigeminal nuclear complex: light and electron microscopic study. J Comp Neurol 2008;507:1521-1541.

26 Ling Z, Chang QA, Tong CW, Leurgans SE, Lipton JW, Carvey PM: Rotenone potentiates dopamine neuron loss in animals exposed to lipopolysaccharide prenatally. Exp Neurol 2004;190:373-383.

27 Lokkegaard A, Nyengaard JR, West MJ: Stereological estimates of number and length of capillaries in subdivisions of the human hippocampal region. Hippocampus 2001;11: 726-740.

28 Gundersen HJG, Jensen EB: The efficiency of systematic sampling in stereology and its prediction. J Microsc 1987;147:229-263.

29 Pakkenberg B, Gundersen HJ: Total number of neurons and glial cells in human brain nuclei estimated by the disector and the fractionator. J Microsc 1988;150:1-20.

30 Champy P, Höglinger GU, Féger J, Gleye C, Hocquemiller R, Laurens A, Guérineau V, Laprévote O, Medja F, Lombès A, Michel PP, Lannuzel A, Hirsch EC, Ruberg M: Annonacin, a lipophilic inhibitor of mitochondrial complex I, induces nigral and striatal neurodegeneration in rats: possible relevance for atypical parkinsonism in Guadeloupe. J Neurochem 2004;88:63-69.
31 Hoglinger GU, Lannuzel A, Khondiker ME, Michel PP, Duyckaerts C, Feger J, Champy P, Prigent A, Medja F, Lombes A, Oertel WH, Ruberg M, Hirsch EC: The mitochondrial complex I inhibitor rotenone triggers a cerebral tauopathy. J Neurochem 2005;95:930939.

32 Chen YR, Chen CL, Zhang L, Green-Church $\mathrm{KB}$, Zweier JL: Superoxide generation from mitochondrial NADH dehydrogenase induces self-inactivation with specific protein radical formation. J Biol Chem 2005;280:3733937348.

33 Fan LW, Tien LT, Zheng B, Pang Y, Rhodes PG, Cai Z: Interleukin-1beta-induced brain injury and neurobehavioral functions in juvenile rats can be attenuated by alpha-phenyl-ntert-butyl-nitrone. Neuroscience 2010;168: 240-252.

34 Schallert T, Woodlee MT: Motor systems: orienting and placing; in Whishaw IQ, Kolb B (eds): The Behaviour of the Laboratory Rat: A Handbook with Tests. New York, Oxford University Press, 2005, pp 129-140.

35 Woodlee MT, Asseo-Garcia AM, Zhao X, Liu SJ, Jones TA, Schallert T: Testing forelimb placing 'across the midline' reveals distinct, lesion-dependent patterns of recovery in rats. Exp Neurol 2005;191:310-317.

- 36 Maciag D, Simpson KL, Coppinger D, Lu Y, Wang Y, Lin RC, Paul IA: Neonatal antidepressant exposure has lasting effects on behavior and serotonin circuitry. Neuropsychopharmacol 2006;31:47-57.

-37 Bartels AL, Leenders KL: Cyclooxygenase and neuroinflammation in Parkinson's disease neurodegeneration. Curr Neuropharmacol 2010;8:62-68.

38 Ruano D, Revilla E, Paz Gavilan M, Vizuete ML, Pintado C, Vitorica J, Castano A: Role of p38 and inducible nitric oxide synthase in the in vivo dopaminergic cells' degeneration induced by inflammatory processes after lipopolysaccharide injection. Neuroscience 2006; 140:1157-1168.

39 Godoy MC, Tarelli R, Ferrari CC, Sarchi MI, Pitossi FJ: Central and systemic IL-1 exacerbates neurodegeneration and motor symptoms in a model of Parkinson's disease. Brain 2008;131:1880-1894.

40 Burke RE: Postnatal developmental programmed cell death in dopamine neurons. Ann NY Acad Sci 2003;991:69-79.

41 Voorn P, Kalsbeek A, Jorritsma-Byham B, Groenewegen HJ: The pre- and post-natal development of the dopaminergic cell groups in the ventral mesencephalon and the dopaminergic innervation of the striatum of the rat. Neuroscience 1988;25:857-887.

42 Costa LG, Aschner M, Vitalone A, Syversen T, Soldin OP: Developmental neuropathology of environmental agents. Annu Rev Pharmacol Toxicol 2004;44:87-110.

43 Godfrey KM, Barker DJ: Fetal programming and adult health. Public Health Nutr 2001;4: 611-624. 
44 Degos V, Favrais G, Kaindl AM, peineau S, Guerrot AM, Verney C, Gressens P: Inflammation processes in perinatal brain damage. J Neural Transm 2010;117:1009-1017.

-45 Miller RL, James-Kracke M, Sun GY, Sun AY: Oxidative and inflammatory pathways in Parkinson's disease. Neurochem Res 2009;34:5565.

46 Uversky V: Neurotoxicant-induced animal models of Parkinson's disease: understanding the role of rotenone, maneb and paraquat in neurodegeneration. Cell Tissue Res 2004;318: 225-241.

-47 Teismann P, Tieu K, Choi DK, Wu DC, Naini A, Hunot S, Vila M, Jackson-Lewis V, Przedborski S: Cyclooxygenase-2 is instrumental in Parkinson's disease neurodegeneration. Proc Natl Acad Sci USA 2003;100:5473-5478.

48 Minghetti L: Cyclooxygenase-2 (COX-2) in inflammatory and degenerative brain diseases. J Neuropathol Exp Neurol 2004;63:901910 .
49 Berg D, Godau J, Riederer P, Gerlach M, Arzberger T: Microglia activation is related to substantia nigra echogenicity. J Neural Transm 2010;117:1287-1292.

50 McGeer PL, Itagaki S, Boyes BE, McGeer EG: Reactive microglia are positive for HLA-DR in the substantia nigra of Parkinson's and Alzheimer's disease brains. Neurology 1988; 38:1285-1291.

51 Gerhard A, Pavese N, Hotton G, Yurheimer F, Es M, Hammers A, Eggert K, Oertel W, Banati RB, Brooks DJ: In vivo imaging of microglial activation with $\left[{ }^{11} \mathrm{C}\right](\mathrm{R})-\mathrm{PK} 11195$ PET in idiopathic Parkinson's disease. Neurobiol Dis 2006;21:404-412.

52 Glass CK, Saijo K, Winner B, Marchetto MC, Gage FH: Mechanisms underlying inflammation in neurodegeneration. Cell 2010;140: 918-934.

53 Qian L, Flood PM, Hong JS: Neuroinflammation is a key player in Parkinson's disease and a prime target for therapy. J Neural Transm 2010;117:971-979.
4 Dutta G, Zhang P, Liu B: The lipopolysaccharide Parkinson's disease animal model: mechanistic studies and drug discovery. Fund Clin Pharmacol 2008;22:453-464.

55 Teismann P, Schulz JB: Cellular pathology of Parkinson's disease: astrocytes, microglia and inflammation. Cell Tissue Res 2004;318:149161.

56 Purisai MG, McComack AL, Cumine S, Li J, Isla MZ, Di Monte DA: Microglial activation as a priming event leading to paraquat-induced dopaminergic cell degeneration. Neurobiol Dis 2007;25:392-400.

57 Sanchez-Guajardo V, Febbraro F, Kirik D, Romero-Ramos M: Microglia acquire distinct activation profiles depending on the degree of a-synuclein neuropathology in a rAAV based model of Parkinson's disease. PLoS One 2010; 5:e8784.

58 Czeh M, Gressens P, Kaindl AM: The yin and yang of microglia. Dev Neurosci 2011;33: 199-209. 